\title{
The lattice of monomial clones on finite fields
}

\author{
Sebastian Kreinecker
}

\begin{abstract}
We investigate the lattice of clones that are generated by a set of functions that are induced on a finite field $\mathbb{F}$ by monomials. We study the atoms and coatoms of this lattice and investigate whether this lattice contains infinite ascending chains, or infinite descending chains, or infinite antichains. We give a connection between the lattice of these clones and semi-affine algebras. Furthermore, we show that the sublattice of idempotent clones of this lattice is finite and every idempotent monomial clone is principal.
\end{abstract}

Mathematics Subject Classification. 08A40, 08A02.

Keywords. Clone, Set of functions, Monomial, Finite field, Semi-affine algebra.

\section{Introduction and preliminaries}

Let $\mathbb{N}:=\{1,2,3, \ldots\}$, let $\mathbb{N}_{0}:=\mathbb{N} \cup\{0\}$, let $A$ be a finite set, let $F \subseteq \bigcup\left\{A^{A^{i}} \mid\right.$ $i \in \mathbb{N}\}$ and let $n \in \mathbb{N}$. We denote $F \cap A^{A^{n}}$ by $F^{[n]}$. Let $C \subseteq \bigcup\left\{A^{A^{i}} \mid i \in \mathbb{N}\right\}$. $C$ is called a clone on $A$ if it is closed under composition of functions, i.e., if $n, m \in$ $\mathbb{N}, f \in C^{[n]}, g_{1}, \ldots, g_{n} \in C^{[m]}$ then $f\left(g_{1}, \ldots, g_{n}\right) \in C^{[m]}$, and if $C$ contains the projections, i.e., for $n, j \in \mathbb{N}$ with $j \leq n, \pi_{j}^{n}: A^{n} \rightarrow A, \pi_{j}^{n}\left(x_{1}, \ldots, x_{n}\right):=x_{j}$ lies in $C$. The characterization of clones on a two-element set by E. Post [13] was the beginning of the study of clones. Already in the case of a three-element set, there are uncountable many clones [6], and thus a full description seems to be hard. Hence the investigation of clones led to the study of clones which contain, or which contain only, specific functions. Results for clones containing only affine mappings are given in [14], a full characterization for polynomial clones (clones containing all the constant functions) on $\mathbb{Z}_{p} \times \mathbb{Z}_{p}$ and on $\mathbb{Z}_{p^{2}}$ for any prime $p$ with the operation + can be found in [4], a description for polynomial clones on $\mathbb{Z}_{p q}$ with the operation + for different primes $p, q$ is given 
in [2], and in [11] it is shown that the lattice of polynomial clones on a group of square-free order that contain the group operation is finite. For general notes on clone theory we refer to [12] and [15].

In this paper we study clones of the following kind: Let $\alpha(1), \alpha(2), \ldots \in$ $\mathbb{N}_{0}$. We call $g=\prod_{i \in \mathbb{N}} x_{i}^{\alpha(i)}$ a monomial if there is an $n \in \mathbb{N}$ such that for all $i \in \mathbb{N}$ with $i>n$ we have $\alpha(i)=0$ and there is a $j \in \mathbb{N}$ such that $\alpha(j) \neq 0$. Then we write $g$ also as $\prod_{i=1}^{n} x_{i}^{\alpha(i)}$. We define $\operatorname{mI}(g)$ by $\max (\{i \in \mathbb{N} \mid \alpha(i) \neq 0\})$ and call $\mathrm{mI}(g)$ the arity of $g$. Let $q=p^{t}$ for some prime $p$ and for some $t \in \mathbb{N}$, and let $\mathbb{F}_{q}:=\operatorname{GF}(q)$. Let $n \geq \operatorname{mI}(g)$. We call the function $g^{\mathbb{F}^{n}}: \mathbb{F}_{q}^{n} \rightarrow \mathbb{F}_{q}$, $g^{\mathbb{F}_{q}^{n}}\left(x_{1}, \ldots, x_{n}\right):=\prod_{i=1}^{n} x_{i}^{\alpha(i)}$ the $n$-ary induced function of $g$ on $\mathbb{F}_{q}$. We call a function which is induced by a monomial a monomial function. We say that $g^{\mathbb{F}^{n}}$ is idempotent if $g^{\mathbb{F}^{n}}(x, \ldots, x)=x$ for all $x \in \mathbb{F}_{q}$, and call then $g$ an idempotent monomial. This holds if and only if $\sum_{i=1}^{\operatorname{mI}(g)} \alpha(i) \equiv_{q-1} 1$. We call two monomials $m_{1}$ and $m_{2}$ equivalent if the $\mathrm{mI}\left(m_{1}\right)$-ary induced function of $m_{1}$ on $\mathbb{F}_{q}$ is equal to the $\mathrm{mI}\left(m_{2}\right)$-ary induced function of $m_{2}$ on $\mathbb{F}_{q}$. Note that $m_{1}=\prod_{i=1}^{n} x_{i}^{\alpha(i)}$ is equivalent to $m_{2}=\prod_{i=1}^{n} x_{i}^{\beta(i)}$ if and only if $\alpha(i) \equiv \beta(i)$ for all $1 \leq i \leq n$, where for $a, b \in \mathbb{N}_{0}$ we let $a \equiv b$ if and only if $a=b$ or $a, b>0$ and $a \equiv_{q-1} b$. Now let $M$ be a set of monomials. We call $M$ a monomial clone on $\mathbb{F}_{q}$ if $C:=\left\{m^{\mathbb{F}_{q}^{n}} \mid m \in M, n \in \mathbb{N}, n \geq \operatorname{mI}(m)\right\}$ is a clone on $\mathbb{F}_{q}$ and $M$ is closed under equivalent monomials, which means if $m \in M$ and some monomial $m^{\prime}$ is equivalent to $m$, then $m^{\prime} \in M$. We say that $M$ is idempotent if all monomials of $M$ are idempotent. For any set $M^{\prime}$ of monomials, there is a least monomial clone $\left\langle M^{\prime}\right\rangle$ containing $M^{\prime}$, the monomial clone on $\mathbb{F}_{q}$ generated by $M^{\prime}$. In order to describe clones that contain only monomial functions, we will describe monomial clones. Clones that contain only monomial functions have been studied in [7], [8], [9], and [10], where special clones (e.g. generated by unary or binary monomial functions) are characterized. This was further developed in [5] where the binary part of an idempotent monomial clone on $\mathbb{F}_{q}$ which is generated by one single binary idempotent monomial is fully described.

Let $\mathbb{M}_{q}$ be the set of all monomial clones on $\mathbb{F}_{q}$. Let $C, D$ be two monomial clones on $\mathbb{F}_{q}$. Then we denote by $C \vee D$ the smallest monomial clone which contains $C$ and $D$, and by $\cap$ we denote the intersection of sets. Then $\left(\mathbb{M}_{q}, \vee, \cap\right)$ is a lattice. The smallest monomial clone $\Delta$ is the monomial clone generated by the monomial $x_{1}$ which induces the clone of projections, and the largest monomial clone $\nabla$ is the monomial clone generated by $x_{1} x_{2}$, which induces the clone generated by the field multiplication. The set of idempotent monomial clones on $\mathbb{F}_{q}$ with $\vee$ and $\cap$ forms a sublattice of the lattice of monomial clones on $\mathbb{F}_{q}$. Let $C$ be a monomial clone on $\mathbb{F}_{q}$ such that $C \neq \Delta$ and $C \neq \nabla$. We call $C$ an atom if for all monomial clones $D$ with $D \subseteq C$ we have $D=\Delta$ or $D=C$. We call $C$ a coatom if for all monomial clones $D$ with $C \subseteq D$ we have $D=\nabla$ or $D=C$. Let $C, D$ be two monomial clones on $\mathbb{F}_{q}$. If $C \subseteq D$ and $C \neq D$, we write $C \subset D$. In this paper we investigate the general structure of the lattice of monomial clones on $\mathbb{F}_{q}$ for any prime power $q$ : In Section 2 we start our investigation on monomials and give techniques for generating monomials. In 
Section 3 we give a full description of the lattice of monomial clones on $\mathbb{F}_{q}$ if $q \in\{2,3,4\}$. In Section 4 we give a connection between monomial clones and semi-affine algebras (see [15]). In Section 5 we investigate the top and the bottom of the lattice of monomial clones on $\mathbb{F}_{q}$ for any prime power $q$. In Theorem 5.4 we see that at the top of the lattice of monomial clones on $\mathbb{F}_{q}$, there is an interval dually isomorphic to the divisor lattice of $q-1$. Furthermore, we get a full description of the atoms in Corollary 5.2 and of the coatoms in Theorem 5.10. In Section 6 we prove that $\left(\mathbb{M}_{q}, \vee, \cap\right)$ is well-partially ordered (Theorem 6.3), i.e., there are no infinite antichains and no infinite descending chains of monomial clones on $\mathbb{F}_{q}$. Furthermore, we show in Theorem 6.8 that infinite ascending chains of monomial clones on $\mathbb{F}_{q}$ exist if and only if $q-1$ is not square-free. In Section 7 we show that the lattice of idempotent monomial clones on $\mathbb{F}_{q}$ is finite and that every idempotent monomial clone on $\mathbb{F}_{q}$ is principal, i.e. singly generated.

\subsection{Notation for monomial clones}

Let $q$ be a prime power. Let $C$ be a clone on $\mathbb{F}_{q}$ which contains only monomial functions, and let $M$ be the monomial clone such that $C=\left\{m^{\mathbb{F}_{q}^{n}} \mid m \in M, n \in\right.$ $\mathbb{N}, n \geq \operatorname{mI}(m)\}$. Let $f \in C$ be induced by the monomial $m=x_{i_{1}}^{\alpha(1)} \cdots x_{i_{n}}^{\alpha(n)}$, where $n \in \mathbb{N}, \alpha(1), \ldots, \alpha(n) \in \mathbb{N}$ and $i_{1}, \ldots, i_{n} \in \mathbb{N}$ with $i_{1}<i_{2}<\cdots<i_{n}$. Then $m$ can be seen as $i_{n}$-ary function and since $m$ has $n$ variables with exponents unequal to 0 , we say that $m$ has width $n$. Since $x^{q}=x$ for all $x \in \mathbb{F}_{q}$ and $M$ is closed under equivalent monomials, we have that $M$ contains all these monomials $x_{i_{1}}^{\beta(1)} \cdots x_{i_{n}}^{\beta(n)}$ where for all $j \leq i_{n}$ we have $\beta(j) \equiv_{q-1} \alpha(j)$ and $\beta(j)>0$. By permuting the variables we have $x_{1}^{\alpha(1)} \cdots x_{n}^{\alpha(n)} \in M$. We see that a monomial clone is uniquely determined by those monomials $\prod_{i=1}^{n} x_{i}^{\alpha(i)}$ with $n \in \mathbb{N}$ such that for all $i \leq n, \alpha(i) \in\{1, \ldots, q-1\}$. Let $i, j \in \mathbb{N}$. If we set a variable $x_{i}$ to a variable $x_{j}$ of a monomial, we say that we identify the variable $x_{i}$ with $x_{j}$. Since $C$ is a clone and $M$ is closed under equivalence of monomials, $M$ is closed under substitution of monomials and thus closed under identifying variables, since a variable induces a projection. Let $M^{\prime}$ be a set of monomials. Then $M^{\prime}$ is a monomial clone on $\mathbb{F}_{q}$ if and only if $\left\{x_{i} \mid i \in \mathbb{N}\right\} \subseteq M^{\prime}, M^{\prime}$ is closed under substitution of monomials and $M^{\prime}$ is closed under equivalent monomials.

Example 1.1. Let $q$ be a prime power. We have $\Delta=\left\langle\left\{x_{1}\right\}\right\rangle$ and $\nabla=\left\langle\left\{x_{1} x_{2}\right\}\right\rangle$. Let $C$ be a monomial clone on $\mathbb{F}_{5}$. If $m\left(x_{1}, x_{2}\right)=x_{1}^{2} x_{2} \in C$, then permuting the variables yields $m_{1}\left(x_{1}, x_{2}, x_{3}\right)=m\left(x_{2}, x_{3}\right)=x_{2}^{2} x_{3} \in C$. Then we have $m_{2}\left(x_{1}, x_{2}, x_{3}\right)=m\left(x_{1}, m_{1}\left(x_{2}, x_{2}, x_{3}\right)\right)=x_{1}^{2} x_{2}^{2} x_{3} \in C$, and therefore we obtain $m_{3}\left(x_{1}, x_{2}, x_{3}, x_{4}\right)=m\left(x_{1}, m_{2}\left(x_{2}, x_{3}, x_{4}\right)\right)=x_{1}^{2} x_{2}^{2} x_{3}^{2} x_{4} \in C$. By identifying all the variables with $x_{1}$ we get that $m_{4}\left(x_{1}\right)=m_{3}\left(x_{1}, x_{1}, x_{1}, x_{1}\right)=x_{1}^{7} \in C$. Since $x_{1}^{7}$ induces the same function as $x_{1}^{3}$, we have $x_{1}^{3} \in C$, and also $x_{1}^{11} \in C$.

Since $x^{q}=x$ for all $x \in \mathbb{F}_{q}$, we will describe monomial clones on $\mathbb{F}_{q}$ by investigating the arithmetic properties modulo $q-1$ of the exponents of the monomials. 


\subsection{Modulus calculation}

Let $q$ be a prime power. Since $x^{q}=x$ for all $x \in \mathbb{F}_{q}$, we calculate modulo $q-1$ in the exponents, but $x^{0} \neq x^{q-1}$ for $x=0$, and thus if an exponent $a$ of a monomial has the property that $a \equiv_{q-1} 0$ and $a>0$, we reduce $a$ to $q-1$ and not to 0 . Hence, we define for $a \in \mathbb{N}_{0}$, its representative with respect to $\equiv$ by

$$
\bar{a}:= \begin{cases}q-1, & \text { if } a \bmod q-1=0 \text { and } a>0, \\ a \bmod q-1, & \text { otherwise. }\end{cases}
$$

If $C$ is a monomial clone on $\mathbb{F}_{q}$, we denote by $\bar{C}$ the set $\left\{\prod_{i=1}^{n} x_{i}^{\alpha(i)} \in C \mid \forall i \leq\right.$ $n: \alpha(i) \in\{1, \ldots, q-1\}\}$. As we already mentioned, $C$ is uniquely determined by $\bar{C}$.

\section{First observations and procedures for generating monomials}

Let $q$ be a prime power and let $C$ be a monomial clone on $\mathbb{F}_{q}$ fixed for the rest of the section. We start with an example of a monomial clone on $\mathbb{F}_{q}$.

Lemma 2.1. Let $b$ be a divisor of $q-1$. The $\operatorname{set} C^{\prime}:=\left\{\prod_{i=1}^{n} x_{i}^{\alpha(i)} \mid n \in \mathbb{N}, \forall i \leq\right.$ $\left.n: \alpha(i) \in \mathbb{N}_{0}, \exists j \leq n: \alpha(j) \neq 0, \sum_{i=1}^{n} \alpha(i) \equiv_{b} 1\right\}$ is a monomial clone on $\mathbb{F}_{q}$.

Proof. Obviously $\left\langle\left\{x_{1}\right\}\right\rangle \subseteq C^{\prime}$. Now we show that $C^{\prime}$ is closed under substitution of monomials. Let $n \in \mathbb{N}$, let $m:=\prod_{i=1}^{n} x_{i}^{\alpha(i)} \in C^{\prime}$, and let $m_{j}:=$ $\prod_{i=1}^{n_{j}} x_{i}^{\beta_{j}(i)} \in C^{\prime}$ for all $j \leq n$. Now we get $m\left(m_{1}, \ldots, m_{n}\right)=\prod_{i=1}^{n} \prod_{i^{\prime}=1}^{n_{i}}$ $x_{i^{\prime}}^{\alpha(i) \cdot \beta_{i}\left(i^{\prime}\right)}$, and we have $\sum_{i=1}^{n} \sum_{i^{\prime}=1}^{n_{i}} \alpha(i) \cdot \beta_{i}\left(i^{\prime}\right) \equiv_{b} \sum_{i=1}^{n} \alpha(i) \equiv_{b} 1$. For all $c, d \in \mathbb{N}$ with $c \equiv_{q-1} d$ we have $c \equiv_{b} d$, since $b$ divides $q-1$, and thus if $\prod_{i=1}^{n} x_{i}^{\alpha(i)} \in C^{\prime}$, then $\left\{\prod_{i=1}^{n} x_{i}^{\beta(i)} \mid \forall i \leq n: \overline{\beta(i)}=\overline{\alpha(i)}\right\} \subseteq C^{\prime}$. Hence, $C^{\prime}$ is a monomial clone on $\mathbb{F}_{q}$.

Lemma 2.2. Let $\prod_{i \in I} x_{i}^{\alpha(i)} \in C$ with $|I| \geq 2$ and $\alpha(i) \neq 0$ for all $i \in I$, and let $D \subset I$ be such that $\sum_{i \in D} \alpha(i) \equiv_{q-1}$ 0. Then $\prod_{i \in I \backslash D} x_{i}^{\alpha(i)} \in C$.

Proof. We identify all $x_{i}$ for $i \in D$ with an $x_{j}$ where $j \in I \backslash D$. The result holds, since $C$ is closed under identifying variables and $\overline{\alpha(j)+\sum_{i \in D} \alpha(i)}=\overline{\alpha(j)}$.

Example 2.3. Let $D$ be a monomial clone on $\mathbb{F}_{5}$. If $x_{1}^{3} x_{2}^{2} x_{3}^{2} \in D$, then $2+2 \equiv_{4} 0$ and thus by Lemma 2.2 we have $x_{1}^{3} \in D$.

Lemma 2.4. Let $t \in \mathbb{N}$, let $\alpha(1), \ldots, \alpha(t) \in \mathbb{N}$ and let $j \in \mathbb{N}$. $C$ contains the monomial $\left(\prod_{i=1}^{t} x_{i}^{\alpha(i)}\right)\left(\prod_{i=t+1}^{t+j} x_{i}^{q-1}\right)$ if and only if for all $n \in \mathbb{N}_{0}$ we have that $C$ contains $\left(\prod_{i=1}^{t} x_{i}^{\alpha(i)}\right)\left(\prod_{i=t+1}^{t+n} x_{i}^{q-1}\right)$.

Proof. The "if"-direction obviously holds. We show the "only if"-direction. By Lemma 2.2, we have $\prod_{i=1}^{t} x_{i}^{\alpha(i)} \in C$. Now we proceed by induction on $n \in \mathbb{N}$. For $n=1$ we have by Lemma 2.2 that $\left(\prod_{i=1}^{t} x_{i}^{\alpha(i)}\right) x_{t+1}^{q-1} \in C$, since $j \in \mathbb{N}$. Now let $n \geq 2$. Let $m\left(x_{1}, \ldots, x_{t+1}\right):=\left(\prod_{i=1}^{t} x_{i}^{\alpha(i)}\right) x_{t+1}^{q-1}$. By the induction 
hypothesis we have that $m^{\prime}\left(x_{1}, \ldots, x_{t+n-1}\right):=\left(\prod_{i=1}^{t} x_{i}^{\alpha(i)}\right)\left(\prod_{i=t+1}^{t+n-1} x_{i}^{q-1}\right) \in$ $C$. Now we have

$$
\begin{aligned}
& m^{\prime}\left(x_{1}, \ldots, x_{t+n-2}, m\left(x_{t+n-1}, x_{t+n}, \ldots, x_{t+n}\right)\right) \\
& \quad=\left(\prod_{i=1}^{t} x_{i}^{\alpha(i)}\right)\left(\prod_{i=t+1}^{t+n-2} x_{i}^{q-1}\right) x_{t+n-1}^{\alpha(1) \cdot(q-1)} x_{t+n}^{\left(\left(\sum_{i=2}^{t} \alpha(i)\right)+(q-1)\right) \cdot(q-1)} \in C,
\end{aligned}
$$

since $C$ is a monomial clone. Therefore $\prod_{i=1}^{t} x_{i}^{\alpha(i)} \prod_{i=t+1}^{t+n} x_{i}^{q-1} \in C$, since $\overline{b \cdot(q-1)}=q-1$ for all $b \in \mathbb{N}$. This finishes the induction step.

Example 2.5. Let $D$ be a monomial clone on $\mathbb{F}_{5}$. If $D$ contains $x_{1}^{2} x_{2}^{3} x_{3}^{4}$, then we get by Lemma 2.4 that $x_{1}^{2} x_{2}^{3} x_{3}^{4} \cdots x_{2+t}^{4} \in D$ for all $t \in \mathbb{N}_{0}$.

Lemma 2.6. Let $N, n \in \mathbb{N}$ with $n \leq N$ and let $f:\{1, \ldots, N\} \rightarrow \mathbb{N}$ be injective. If $C$ contains $\prod_{i=1}^{N} x_{f(i)}^{\alpha(i)}$, then there exists $\gamma \in \mathbb{N}_{0}$ such that $C$ contains $x_{1}^{\alpha(1)} \cdots x_{n}^{\alpha(n)} x_{n+1}^{\gamma}$.

Proof. For all $i \in\{1, \ldots, n\}$ we substitute the variable $x_{i}$ for the variable $x_{f(i)}$, and for all $i \in\{n+1, \ldots, N\}$ we substitute the variable $x_{n+1}$ for $x_{f(i)}$.

Lemma 2.7. Let $t \in \mathbb{N}_{0}$ and let $\alpha(1), \ldots, \alpha(t) \in \mathbb{N}$. If $x_{1} \prod_{i=1}^{t} x_{i+1}^{\alpha(i)} \in C$, then we have for all $n \in \mathbb{N}_{0}$ that $x_{1} \prod_{j=0}^{n-1} \prod_{i=1}^{t} x_{j \cdot t+i+1}^{\alpha(i)} \in C$.

Proof. Let $m\left(x_{1}, \ldots, x_{t+1}\right):=x_{1} \prod_{i=1}^{t} x_{i+1}^{\alpha(i)}$. We proceed by induction on $n$. The statement is true for $n=0$, since $x_{1} \in C$ and for $n=1$, since $m \in C$. Let $n>1$. By the induction hypothesis, we have $m^{\prime}\left(x_{1}, \ldots, x_{(n-1) t+1}\right):=$ $x_{1} \prod_{j=0}^{n-2} \prod_{i=1}^{t} x_{j \cdot t+i+1}^{\alpha(i)} \in C$. Now we have

$$
\begin{aligned}
m^{\prime \prime}\left(x_{1}, \ldots, x_{n \cdot t+1}\right) & :=m^{\prime}\left(m\left(x_{1}, x_{(n-1) t+1+1}, \ldots, x_{n \cdot t+1}\right), x_{2}, \ldots, x_{(n-1) t+1}\right) \\
& =x_{1}\left(\prod_{i=1}^{t} x_{(n-1) t+i+1}^{\alpha(i)}\right) \prod_{j=0}^{n-2} \prod_{i=1}^{t} x_{j \cdot t+i+1}^{\alpha(i)} \\
& =x_{1} \prod_{j=0}^{n-1} \prod_{i=1}^{t} x_{j \cdot t+i+1}^{\alpha(i)},
\end{aligned}
$$

which lies in $C$, since $C$ is a monomial clone. This finishes the induction step.

We will often use Lemma 2.7 in the following context:

Example 2.8. Let $k \in \mathbb{N}$. If $x_{1} \cdots x_{1+k} \in C$, then we get by Lemma 2.7 that for all $t \in \mathbb{N}$ we have $x_{1} \cdots x_{1+t \cdot k} \in C$.

Lemma 2.9. If $C$ contains a monomial with two times the exponent 1 , then $x_{1} \cdots x_{q} \in C$.

Proof. By Lemma 2.6 we have $m=x_{1} x_{2} x_{3}^{\alpha} \in C$ for some $\alpha \in \mathbb{N}_{0}$. Now we get by Lemma 2.7 that $x_{1}\left(x_{2} x_{3}^{\alpha}\right)\left(x_{2+2} x_{3+2}^{\alpha}\right) \cdots\left(x_{2+(q-2) 2} x_{3+(q-2) 2}^{\alpha}\right) \in C$. 
By renaming the variables we get $x_{1} x_{2} \cdots x_{q} x_{q+1}^{\alpha} \cdots x_{2(q-1)+1}^{\alpha} \in C$. By identifying the variables from $x_{q+2}$ to $x_{2(q-1)+1}$ with the variable $x_{q+1}$ we get $x_{1} x_{2} \cdots x_{q} x_{q+1}^{(q-1) \cdot \alpha} \in C$. The result follows now from Lemma 2.2.

Lemma 2.10. We assume that $C$ contains $x_{1}^{\alpha(1)} \cdots x_{n}^{\alpha(n)}$ where $n \in \mathbb{N}$ with $n \geq 2, \operatorname{gcd}(\alpha(1), q-1)=1, \alpha(1), \alpha(2) \in \mathbb{N}, \alpha(i) \in \mathbb{N}_{0}$ for $i>2$. Then there exists $\gamma \in \mathbb{N}_{0}$ such that $x_{1} x_{2}^{\alpha(2)} \cdots x_{n}^{\alpha(n)} x_{n+1}^{\gamma} \in C$.

Proof. If $\alpha(1)=1$ the result is obvious. Now we assume that $\alpha(1) \neq 1$. Let $m\left(x_{1}, \ldots x_{n}\right):=x_{1}^{\alpha(1)} \cdots x_{n}^{\alpha(n)} \in C$. We show by induction that for all $t \in \mathbb{N}$, there is a $\gamma \in \mathbb{N}_{0}$ such that $x_{1}^{\alpha(1)^{t}} x_{2}^{\alpha(2)} \cdots x_{n}^{\alpha(n)} x_{n+1}^{\gamma} \in C$. For $t=1$, we have $m \in C$. Let $t>1$. By the induction hypothesis, we have $m^{\prime}\left(x_{1}, \ldots, x_{n+1}\right)=$ $x_{1}^{\alpha(1)^{(t-1)}} x_{2}^{\alpha(2)} \cdots x_{n}^{\alpha(n)} x_{n+1}^{\gamma} \in C$ for some $\gamma \in C$. Now we get

$$
\begin{aligned}
m^{\prime \prime}\left(x_{1}, \ldots, x_{n+1}\right) & :=m\left(m^{\prime}\left(x_{1}, x_{n+1} \ldots, x_{n+1}\right), x_{2}, \ldots, x_{n}\right) \\
& =x_{1}^{\alpha(1)^{t}} x_{2}^{\alpha(2)} \cdots x_{n}^{\alpha(n)} x_{n+1}^{\gamma^{\prime}} \in C,
\end{aligned}
$$

where $\gamma^{\prime}=\alpha(1) \cdot\left(\gamma+\sum_{i=2}^{k} \alpha(i)\right)$. This concludes the induction step. If $q-1=1$, let $t=1$ and then $\overline{\alpha(1)^{t}}=\overline{\alpha(1)}=1$, since $\alpha(1)>0$. If $q-1>1$, then let $t=\phi(q-1)$, where $\phi$ denotes Euler's totient function. Then $\overline{\alpha(1)^{t}}=1$, because $\operatorname{gcd}(\alpha(1), q-1)=1$. Since $C$ is closed under equivalent monomials, we get $x_{1} x_{2}^{\alpha(2)} \cdots x_{n}^{\alpha(n)} x_{n+1}^{\gamma^{\prime}} \in C$.

Lemma 2.11. Let $n \in \mathbb{N}, n \geq 2$ and let $\alpha(1), \ldots, \alpha(n) \in \mathbb{N}$ with $\operatorname{gcd}(\alpha(1), q-$ $1)=\operatorname{gcd}(\alpha(2), q-1)=1$. If $C$ contains $x_{1}^{\alpha(1)} x_{2}^{\alpha(2)} \cdots x_{n}^{\alpha(n)}$, then $x_{1} \cdots x_{q} \in C$.

Proof. By Lemma 2.10 and Lemma 2.6 we get $x_{1} x_{2}^{\alpha(2)} x_{3}^{\gamma} \in C$ for some exponent $\gamma \in \mathbb{N}_{0}$. Since $C$ is a monomial clone, we have $x_{1}^{\alpha(2)} x_{2} x_{3}^{\gamma} \in C$ and thus we get by Lemma 2.10 and Lemma 2.6 that $x_{1} x_{2} x_{3}^{\delta} \in C$ for some $\delta \in \mathbb{N}_{0}$. The result follows now from Lemma 2.9.

Lemma 2.12. Let $k \in \mathbb{N}$ and let $\alpha \in\{1, \ldots, q\}$. We assume that $x_{1} \cdots x_{k} \in C$ and $x_{1}^{\alpha} \in C$. If $k>q-\alpha$, then $x_{1} \cdots x_{k-(q-\alpha)} \in C$.

Proof. We assume that $\alpha<q$, otherwise the claim is trivial. Let $t:=q-1-\alpha$ and let $m\left(x_{1}, \ldots, x_{k}\right):=x_{1} \cdots x_{k}$. Since $k-(q-\alpha)>0$, we have that

$$
\begin{aligned}
m^{\prime}\left(x_{1}, \ldots, x_{k-t+1}\right) & :=m\left(x_{1}, \ldots, x_{k-t}, x_{k-t+1}, \ldots, x_{k-t+1}\right) \\
& =x_{1} \cdots x_{k-t} x_{k-t+1}^{t}
\end{aligned}
$$

lies in $C$. Since $x_{1}^{\alpha} \in C$, we get by substitution of monomials that

$$
\begin{aligned}
m^{\prime \prime}\left(x_{1}, \ldots, x_{k-t+1}\right) & :=m^{\prime}\left(x_{1}, \ldots, x_{k-t-1}, x_{k-t}^{\alpha}, x_{k-t+1}\right) \\
& =x_{1} \cdots x_{k-t-1} x_{k-t}^{\alpha} x_{k-t+1}^{t} \in C .
\end{aligned}
$$

Finally, we get $m^{\prime \prime}\left(x_{1}, \ldots, x_{k-t-1}, x_{k-t-1}, x_{k-t-1}\right)=x_{1} \cdots x_{k-t-1}^{1+t+\alpha} \in C$. Then $x_{1} \cdots x_{k-(q-\alpha)} \in C$, since $\overline{1+t+\alpha}=1$ and $1+t=q-\alpha$, and thus $k-t-1=$ $k-(q-\alpha)$. 
As a special case we get the following corollary:

Corollary 2.13. We assume that $C$ contains $x_{1} \cdots x_{q}$. Let $\alpha \in\{1, \ldots, q\}$ such that $x_{1}^{\alpha} \in C$. Then $x_{1} \cdots x_{\alpha} \in C$.

Proof. By Lemma 2.12 we have $x_{1} \cdots x_{q-(q-\alpha)}=x_{1} \cdots x_{\alpha} \in C$.

Lemma 2.14. $\left\langle\left\{x_{1} \cdots x_{q}\right\}\right\rangle$ is the clone of all idempotent monomials over $\mathbb{F}_{q}$.

Proof. We have $x_{1} \cdots x_{q}=x_{1} \prod_{i=1}^{q-1} x_{i+1}$. By Lemma 2.7 we have for each $n \in \mathbb{N}$ that

$$
x_{1} \cdots x_{q} x_{q+1} \cdots x_{q+n(q-1)} \in\left\langle\left\{x_{1} \cdots x_{q}\right\}\right\rangle,
$$

and thus we can generate all monomials with total degree 1 modulo $q-1$ by identifying and permuting variables. Hence, $\left\langle\left\{x_{1} \cdots x_{q}\right\}\right\rangle$ contains all idempotent monomials over $\mathbb{F}_{q}$. On the other hand, the clone of all idempotent monomial contains $x_{1} \cdots x_{q}$.

More generally:

Lemma 2.15. Let $k \in \mathbb{N} \backslash\{1\}$. Then $\left\langle\left\{x_{1} \cdots x_{k}\right\}\right\rangle$ consists of all monomials $x_{1}^{\alpha(1)} \cdots x_{n}^{\alpha(n)}$ with $\sum_{i=1}^{n} \alpha(i) \equiv_{\operatorname{gcd}(k-1, q-1)} 1$.

Proof. By Lemma 2.11 we have $x_{1} \cdots x_{q} \in C:=\left\langle\left\{x_{1} \cdots x_{k}\right\}\right\rangle$. By identifying the variables $x_{3}, \ldots, x_{k}$ with $x_{2}$ of the monomial $x_{1} \cdots x_{k}$, we get that $x_{1} x_{2}^{k-1} \in$ $C$. By Lemma 2.7 and by identifying variables, we have for all $t \in \mathbb{N}$ that $x_{1} x_{2}^{t(k-1)} \in C$. There are $t_{1}, t_{2} \in \mathbb{Z}$ such that $t_{1} \cdot(k-1)+t_{2} \cdot(q-1)=\operatorname{gcd}(k-$ $1, q-1)$, and thus there is a $t^{\prime} \in \mathbb{N}$ such that $\overline{t^{\prime} \cdot(k-1)}=\operatorname{gcd}(k-1, q-1)$. This means that $x_{1} x_{2}^{\operatorname{gcd}(k-1, q-1)} \in C$, and by identifying variables we get $x_{1}^{1+\operatorname{gcd}(k-1, q-1)} \in C$. Since $x_{1} \cdots x_{q} \in C$ and $x_{1}^{1+\operatorname{gcd}(k-1, q-1)} \in C$, we get by Corollary 2.13 that $x_{1} \cdots x_{1+\operatorname{gcd}(k-1, q-1)} \in C$. By Lemma 2.7 we get now for all $n \in \mathbb{N}$ that $x_{1} \cdots x_{1+n \cdot \operatorname{gcd}(k-1, q-1)} \in C$. Hence, we can generate all monomials where the sum of the exponents is congruent to $1 \operatorname{modulo} \operatorname{gcd}(k-1, q-1)$ by identifying variables.

Finally, the set of such monomials is a monomial clone by Lemma 2.1, and it clearly contains $x_{1} \cdots x_{k}$.

\section{The whole lattice of monomial clones if $q \leq 4$}

We start our investigation on the lattice of monomial clones for the cases $\mathbb{F}_{2}$ and $\mathbb{F}_{3}$. The following Proposition slightly generalizes the result of [9] and [7, Corollary 3.7] for $\mathbb{F}_{3}$ where the lattice of monomial clones which are generated by a single monomial is given. In the case of $\mathbb{F}_{2}$ these two lattices are equal and in the case of $\mathbb{F}_{3}$ the difference of the number of members of these two lattices is one, which means, there is exactly one monomial clone on $\mathbb{F}_{3}$ that is not singly generated.

Proposition 3.1. The lattices of monomial clones on $\mathbb{F}_{2}$ and $\mathbb{F}_{3}$ are given in Figure 1. 


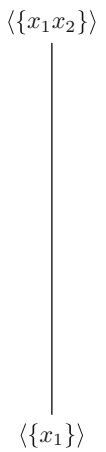

(A) Lattice of monomial clones on $\mathbb{F}_{2}$

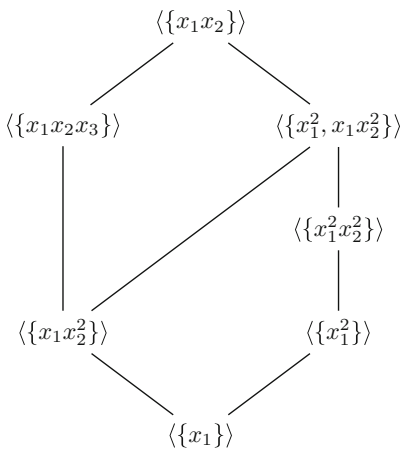

(B) Lattice of monomial clones on $\mathbb{F}_{3}$

Figure 1. Monomial Clones on $\mathbb{F}_{2}$ and $\mathbb{F}_{3}$

Proof. Let $C$ be a monomial clone on $\mathbb{F}_{2}$. All functions on $\mathbb{F}_{2}$ can be induced by polynomials with only 0 and 1 as exponents. There is only one unary function that is induced by a monomial on $\mathbb{F}_{2}$, namely the function induced by $x_{1}$. If $C$ contains a monomial with width $t \in \mathbb{N}$ with $t>1$, then $C$ contains $x_{1} \ldots x_{t}$. The result follows now from Lemma 2.4.

Let us now determine the monomial clones on $\mathbb{F}_{3}: C_{1}=\left\langle\left\{x_{1}\right\}\right\rangle$ is the smallest monomial clone. $C_{2}=\left\langle\left\{x_{1}^{2}\right\}\right\rangle$ contains all monomials of width 1 , and thus $C_{1} \subset C_{2}$ and there are no other monomial clones between $C_{1}$ and $C_{2}$, since $\bar{a} \in\{0,1,2\}$ for all $a \in \mathbb{N}_{0}$. Let $C_{3}:=\left\langle\left\{x_{1}^{2} x_{2}^{2}\right\}\right\rangle$. By Lemma 2.2 and the fact that $C_{2}$ only contains monomials of width 1 we have $C_{2} \subset C_{3}$. By Lemma 2.4 we have that $C_{3}$ contains all monomials $x_{1}^{2} \cdots x_{n}^{2}$ with $n \in \mathbb{N}$. By Lemma 2.4 every monomial in $C_{3}$ of width greater 1 generates $C_{3}$, and therefore there are no other monomial clones between $C_{2}$ and $C_{3}$. Now let $C$ be a monomial clone which contains a monomial that does not lie in $\left\langle\left\{x_{1}\right\}\right\rangle$ but contains 1 as exponent. Then $C$ contains $m=x_{1} \prod_{i=2}^{n} x_{i}^{\alpha(i)}$ for $n \geq 2$ and $\alpha(2), \ldots, \alpha(n) \in\{1,2\}$. First, we assume that $1+|\{j \in\{2, \ldots, n\} \mid \alpha(j)=1\}|$ is even. Then by Lemma 2.2,C contains the monomial $x_{1} x_{2}$ and thus all monomials. Now we assume that $1+|\{j \in\{2, \ldots, n\} \mid \alpha(j)=1\}|$ is odd and $C$ does not contain a monomial with an even number $(>0)$ of 1 's as exponent. If $1+|\{j \in\{2, \ldots, n\} \mid \alpha(j)=1\}|=1$, we have $m=x_{1} \prod_{i=2}^{n} x_{i}^{2}$. By Lemma 2.2 we have $x_{1} x_{2}^{2} \in C$ and by Lemma 2.4 we have $m \in\left\langle\left\{x_{1} x_{2}^{2}\right\}\right\rangle$. We see that $\overline{\left\langle\left\{x_{1} x_{2}^{2}\right\}\right\rangle}$ contains exactly all monomials with exactly one exponent with 1 and thus $x_{1}^{2} \notin\left\langle\left\{x_{1} x_{2}^{2}\right\}\right\rangle$. If $x_{1}^{2} \in C$, we get $x_{1}^{2} x_{2}^{2} \in C$ by plugging $x_{1}^{2}$ into $x_{1}$ of $x_{1} x_{2}^{2}$. If $x_{1}^{2} \cdots x_{n^{\prime}}^{2} \in C$ for some $n^{\prime} \geq 2$ we get by Lemma 2.2 that $x_{1}^{2} \in C$ and thus there are no monomial clones between $\left\langle\left\{x_{1} x_{2}^{2}\right\}\right\rangle$ and $\left\langle\left\{x_{1}^{2}, x_{1} x_{2}^{2}\right\}\right\rangle$, and neither between $\left\langle\left\{x_{1}^{2} x_{2}^{2}\right\}\right\rangle$ and $\left\langle\left\{x_{1}^{2}, x_{1} x_{2}^{2}\right\}\right\rangle$. Now we assume $1+|\{j \in\{2, \ldots, n\} \mid \alpha(j)=1\}|>1$. Then, by Lemma 2.2, $C$ contains the monomial $x_{1} x_{2} x_{3}$. We see that $x_{1} x_{2} x_{3}$ does not generate $x_{1}^{2}$, since $\overline{\left\langle\left\{x_{1} x_{2} x_{3}\right\}\right\rangle}$ contains only monomials with an odd number of variables with exponent 1. 


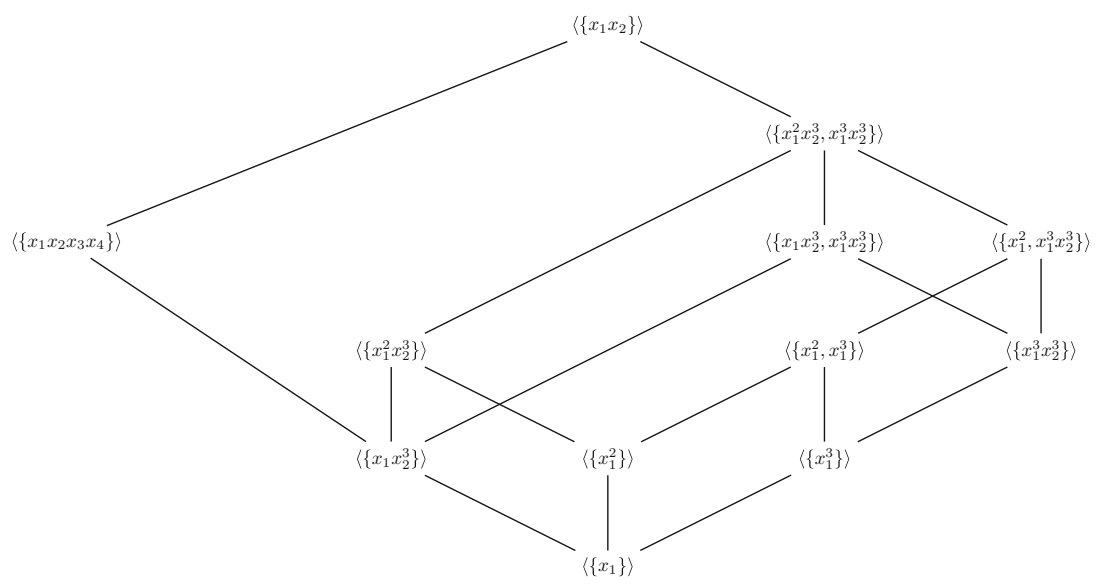

FiguRE 2. Lattice of monomial clones on $\mathbb{F}_{4}$

We also have $\left\langle\left\{x_{1} x_{2}^{2}\right\}\right\rangle \subset\left\langle\left\{x_{1} x_{2} x_{3}\right\}\right\rangle$, since all monomials of $\overline{\left\langle\left\{x_{1} x_{2}^{2}\right\}\right\rangle}$ contain exactly one exponent with 1 . If $C$ contains $x_{1} x_{2} x_{3}$ and $x_{1}^{2}$, then $C$ contains $x_{1} x_{2} x_{3}^{2}$ and Lemma 2.2 yields $x_{1} x_{2} \in C$. This finishes the proof.

The next proposition describes the whole lattice of monomial clones if $q=4$.

Proposition 3.2. The lattice of monomial clones on $\mathbb{F}_{4}$ is given in Figure 2.

Proof. Let $C$ be a monomial clone on $\mathbb{F}_{4}$. If $C$ contains $x_{1} x_{2}$, then $C$ contains all monomials. First, we show that $\left\langle\left\{x_{1} x_{2} x_{3} x_{4}\right\}\right\rangle$ and $\left\langle\left\{x_{1} x_{2}\right\}\right\rangle$ are different and that these two monomial clones are the only monomial clones that contain a monomial which contains two exponents different from 0 modulo 3 . To this end, we assume that $C$ contains a monomial $m=x_{1}^{\alpha(1)} x_{2}^{\alpha(2)} \cdots x_{n}^{\alpha(n)}$ where $n \in \mathbb{N} \backslash\{1\}$ and at least 2 exponents are not equal to 0 modulo 3 . We assume that $\alpha(1) \not z_{3} 0$ and $\alpha(2) \not_{3} \quad 0$. Then Lemma 2.11 yields $x_{1} x_{2} x_{3} x_{4} \in C$, since $\operatorname{gcd}(\alpha(1), 3)=1$ and $\operatorname{gcd}(\alpha(2), 3)=1$. By Lemma $2.14 C$ contains all idempotent monomials. Now we assume that $x_{1} \cdots x_{4} \in C$ and $C$ contains a monomial $m^{\prime}$ which is not idempotent. By identifying all variables of $m^{\prime}$ with $x_{1}$, we get that there exists $\alpha \in \mathbb{N}$ such that $C$ contains $x_{1}^{\alpha}$. Since $m^{\prime}$ is not idempotent, we have $\bar{\alpha} \neq 1$. If $\bar{\alpha}=3$, we have $x_{1} x_{2} x_{3} x_{4}, x_{1}^{3} \in C$ and thus $x_{1} x_{2} x_{3}^{3} x_{4}^{3} \in C$. Now Lemma 2.2 yields $x_{1} x_{2} \in C$. If $\bar{\alpha}=2$, then $C$ contains $x_{1}^{2}$. This means that $x_{1} x_{2} x_{3} x_{4}, x_{1}^{2} \in C$ and thus Corollary 2.13 yields $x_{1} x_{2} \in C$. If $C$ contains only idempotent monomials, then we get by Lemma 2.14 that $C=\left\langle\left\{x_{1} x_{2} x_{3} x_{4}\right\}\right\rangle$ and $x_{1} x_{2} \notin C$. By the previous analysis we have, if $C$ contains a monomial which is not idempotent and it contains two exponents which are not 0 modulo 3 , then $C$ contains $x_{1} x_{2}$.

Now we assume that all monomials of $C$ contain at most one exponent which is not equal to 0 modulo 3 . By Lemma 2.2 and Lemma 2.4 we can restrict to the following monomials as possible generators for $C: x_{1}^{2}, x_{1}^{3}, x_{1} x_{2}^{3}$, 
$x_{1}^{2} x_{2}^{3}$, and $x_{1}^{3} x_{2}^{3}$. These monomials induce different functions. Now we want to find all monomial clones which we get by different combinations of these generators. We start with different combinations including $x_{1}^{3} x_{2}^{3}$. We have that $x_{1}^{2} \in\left\langle\left\{x_{1}^{2} x_{2}^{3}\right\}\right\rangle, x_{1}^{3} \in\left\langle\left\{x_{1}^{3} x_{2}^{3}\right\}\right\rangle$ and $x_{1} x_{2}^{3} \in\left\langle\left\{x_{1}^{2} x_{2}^{3}\right\}\right\rangle$. Hence, the monomial clones above $\left\langle\left\{x_{1}^{3} x_{2}^{3}\right\}\right\rangle$ and below $\left\langle\left\{x_{1} x_{2}\right\}\right\rangle$ are given by $\left\langle\left\{x_{1} x_{2}^{3}, x_{1}^{3} x_{2}^{3}\right\}\right\rangle$, $\left\langle\left\{x_{1}^{2}, x_{1}^{3} x_{2}^{3}\right\}\right\rangle$, and $\left\langle\left\{x_{1}^{2} x_{2}^{3}, x_{1}^{3} x_{2}^{3}\right\}\right\rangle$. We have that $x_{1}^{2} \notin\left\langle\left\{x_{1} x_{2}^{3}, x_{1}^{3} x_{2}^{3}\right\}\right\rangle$, since $x_{1}^{2}$ does not preserve the set $\{1, a\}$ where $a$ is a generator for $\left(\mathbb{F}_{4} \backslash\{0\}, \cdot\right)$, but the operations on $\mathbb{F}_{4}$ given by $\left(x_{1}, x_{2}\right) \mapsto x_{1} x_{2}^{3}$ and $\left(x_{1}, x_{2}\right) \mapsto x_{1}^{3} x_{2}^{3}$ preserve the set $\{1, a\}$. On the other hand we have that $x_{1} x_{2}^{3} \notin\left\langle\left\{x_{1}^{2}, x_{1}^{3} x_{2}^{3}\right\}\right\rangle$, since all monomials of $\left\langle\left\{x_{1}^{2}, x_{1}^{3} x_{2}^{3}\right\}\right\rangle$ of width larger than 1 have the property that all exponents are 0 modulo 3 . Therefore, $\left\langle\left\{x_{1} x_{2}^{3}, x_{1}^{3} x_{2}^{3}\right\}\right\rangle \not \supset x_{1}^{2}$ and $\left\langle\left\{x_{1}^{2}, x_{1}^{3} x_{2}^{3}\right\}\right\rangle \not \supset x_{1} x_{2}^{3}$ are distinct and both monomial clones are strictly contained in $\left\langle\left\{x_{1}^{2} x_{2}^{3}, x_{1}^{3} x_{2}^{3}\right\}\right\rangle$. Then the combinations of the generators $x_{1}^{2}, x_{1}^{3}, x_{1} x_{2}^{3}, x_{1}^{2} x_{2}^{3}$ are left. Now we try to find different monomial clones if $x_{1}^{2} x_{2}^{3}$ is a generator. We have $x_{1}^{2} \in\left\langle\left\{x_{1}^{2} x_{2}^{3}\right\}\right\rangle$, $x_{1} x_{2}^{3} \in\left\langle\left\{x_{1}^{2} x_{2}^{3}\right\}\right\rangle$, and if $x_{1}^{3}$ and $x_{1}^{2} x_{2}^{3}$ lie in $C$, then $x_{1}^{3} x_{2}^{3} \in C$. Hence, we just get the monomial clone $\left\langle\left\{x_{1}^{2} x_{2}^{3}\right\}\right\rangle$ as a new one. Now the combinations of the generators $x_{1}^{2}, x_{1}^{3}, x_{1} x_{2}^{3}$ are left. If $x_{1}^{3} \in C$ and $x_{1} x_{2}^{3} \in C$, then $x_{1}^{3} x_{2}^{3} \in C$, and if $x_{1}^{2} \in C$ and $x_{1} x_{2}^{3} \in C$, then $x_{1}^{2} x_{2}^{3} \in C$. Hence, we only get the monomial clones $\left\langle\left\{x_{1} x_{2}^{3}\right\}\right\rangle,\left\langle\left\{x_{1}^{2}\right\}\right\rangle,\left\langle\left\{x_{1}^{3}\right\}\right\rangle$ and $\left\langle\left\{x_{1}^{2}, x_{1}^{3}\right\}\right\rangle$, which we have not found yet. The smallest monomial clone is given by $\left\langle\left\{x_{1}\right\}\right\rangle$. Altogether we have found 12 different monomial clones, which are ordered as given in Figure 2.

\section{Connection to semi-affine algebras}

In this section we give a connection of monomial clones to semi-affine algebras. First, we recall the definition of a semi-affine algebra (cf. [15]). Let $\mathbf{A}=$ $(A,+,-, 0)$ be an abelian group. Let $n \in \mathbb{N}_{0}$ and let $f$ be an $n$-ary operation on $A$. We call $f$ affine with respect to $\mathbf{A}$ if $f\left(u+{ }^{\prime} v\right)+f(0, \ldots, 0)=f(u)+f(v)$ for all $u, v \in A^{n}$, where ${ }^{+}{ }^{\prime}$ is the componentwise addition. We call then an algebra $\mathbf{B}=(A, F)$ semi-affine with respect to $\mathbf{A}$ if every $f \in F$ is affine with respect to $\mathbf{A}$. Furthermore, we say that $f$ is 0 -preserving if $f(0, \ldots, 0)=0$. Let $F \subseteq \bigcup\left\{A^{A^{i}} \mid i \in \mathbb{N}\right\}$. We call the algebra $(A, F) 0$-preserving if all term functions of $(A, F)$ are 0 -preserving. Let $C$ be a clone on $A$. We call $C$ 0-preserving semi-affine with respect to $\mathbf{A}$ if $(A, C)$ is a 0 -preserving and semi-affine algebra with respect to $\mathbf{A}$. For $a \in \mathbb{N}_{0}$, we write $[a] \in \mathbb{Z}_{q-1}$ for the equivalence class of $a$ modulo $q-1$. Let $q$ be a prime power and let $G$ be a set of monomials. Then we define

$\varphi(G):=\left\{f: \mathbb{Z}_{q-1}^{n} \rightarrow \mathbb{Z}_{q-1},\left(y_{1}, \ldots, y_{n}\right) \mapsto \sum_{i=1}^{n}[r(i)] y_{i} \mid n \in \mathbb{N}, \prod_{i=1}^{n} x_{i}^{r(i)} \in G\right\}$.

If $G$ is a monomial clone on $\mathbb{F}_{q}$, then the set $\varphi(G)$ is a 0 -preserving semi-affine clone with respect to $\left(\mathbb{Z}_{q-1},+,-, 0\right)$. Note that for all $C, D \in \mathbb{M}_{q}$ with $C \subseteq D$ we have $\varphi(C) \subseteq \varphi(D)$. On the other hand we have that the monomial clones $\varphi\left(\left\langle\left\{x_{1}^{q-1}\right\}\right\rangle\right)$ and $\varphi\left(\left\langle\left\{x_{1}^{q-1} x_{2}^{q-1}\right\}\right\rangle\right)$ are both equal to the clone on $\mathbb{Z}_{q-1}$ that is generated by $\{0\}$, but $\left\langle\left\{x_{1}^{q-1}\right\}\right\rangle \subset\left\langle\left\{x_{1}^{q-1} x_{2}^{q-1}\right\}\right\rangle$. Let $F$ be a set of finitary 


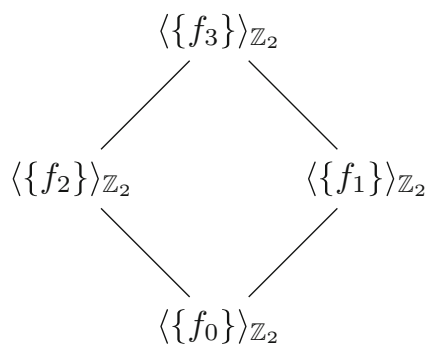

FiguRE 3. Lattice of 0-preserving semi-affine clones with respect to $\left(\mathbb{Z}_{2},+,-, 0\right)$

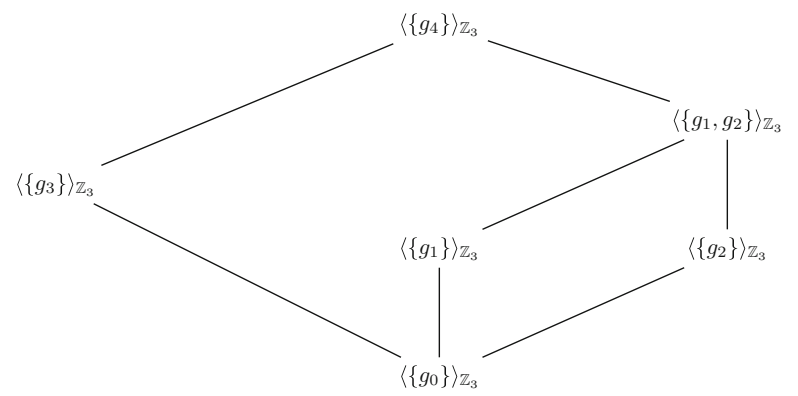

FiguRE 4. Lattice of 0-preserving semi-affine clones with respect to $\left(\mathbb{Z}_{3},+,-, 0\right)$

operations on $\mathbb{Z}_{q-1}$. We denote the clone generated by $F$ by $\langle F\rangle_{\mathbb{Z}_{q-1}}$. Let us consider the difference of monomial clones and semi-affine algebras with respect to $\left(\mathbb{Z}_{q-1},+,-, 0\right)$ in the example $q=3$. Let $f_{0}: \mathbb{Z}_{2} \rightarrow \mathbb{Z}_{2}, x \mapsto x, f_{1}: \mathbb{Z}_{2} \rightarrow$ $\mathbb{Z}_{2}, x \mapsto 0, f_{2}: \mathbb{Z}_{2}^{3} \rightarrow \mathbb{Z}_{2},(x, y, z) \mapsto x+y+z$, and $f_{3}: \mathbb{Z}_{2}^{2} \rightarrow \mathbb{Z}_{2},(x, y) \mapsto x+y$ Then the lattice of 0 -preserving semi-affine clones with respect to $\left(\mathbb{Z}_{2},+,-, 0\right)$ is given by Figure 3 .

In Proposition 3.1 we find the lattice of monomial clones on $\mathbb{F}_{3}$ and see that $\left|\mathbb{M}_{3}\right|=7$. We will give a connection between monomial clones on $\mathbb{F}_{q}$ and 0 -preserving semi-affine algebras with respect to $\left(\mathbb{Z}_{q-1},+,-, 0\right)$ via $\varphi$ in Proposition 4.3, which we use in Section 6 and Section 7. If $q=4$ we have given the lattice of monomial clones in Theorem 3.2. From this lattice we can easily derive the lattice of 0 -preserving semi-affine clones with respect to $\left(\mathbb{Z}_{3},+,-, 0\right)$ given in Figure 4 . For this figure, we use $g_{0}: \mathbb{Z}_{3} \rightarrow \mathbb{Z}_{3}, x \mapsto x$, $g_{1}: \mathbb{Z}_{3} \rightarrow \mathbb{Z}_{3}, x \mapsto 0, g_{2}: \mathbb{Z}_{3} \rightarrow \mathbb{Z}_{3}, x \mapsto 2 x, g_{3}: \mathbb{Z}_{3}^{4} \rightarrow \mathbb{Z}_{3},\left(x_{1}, x_{2}, x_{3}, x_{4}\right) \mapsto$ $x_{1}+x_{2}+x_{3}+x_{4}$, and let $g_{4}: \mathbb{Z}_{3}^{2} \rightarrow \mathbb{Z}_{3},(x, y) \mapsto x+y$.

Now we investigate some properties of the lattice of monomial clones if $q-1$ is square-free.

Lemma 4.1. Let $q$ be a prime power such that $q-1$ is square-free, let $n \in$ $\mathbb{N}_{0}$ and let $d, \alpha(1), \ldots, \alpha(n) \in \mathbb{N}$. Let $C$ be a monomial clone on $\mathbb{F}_{q}$. If $x_{1}^{d} x_{2}^{d} \prod_{i=1}^{n} x_{2+i}^{\alpha(i)} \in C$, then $x_{1}^{d} x_{2}^{d}\left(\prod_{i=1}^{n} x_{2+i}^{\alpha(i)}\right) x_{3+n}^{q-1} \in C$. 
Proof. Let $m\left(x_{1}, \ldots, x_{n+2}\right):=x_{1}^{d} x_{2}^{d} \prod_{i=1}^{n} x_{2+i}^{\alpha(i)}$. We show by induction that for all $k \in \mathbb{N}$, we have

$$
m_{k}=\left(\prod_{j=1}^{k} x_{j}^{d^{j}}\right) x_{k+1}^{d^{k}}\left(\prod_{j=1}^{k} \prod_{i=1}^{n} x_{(j-1) n+i+k+1}^{d^{k-j} \cdot \alpha(i)}\right) \in C
$$

For $k=1$, we have $m_{1}=m \in C$. Now we assume that $k>1$ and the induction hypothesis holds for $k-1$. This means

$m_{k-1}\left(x_{1}, \ldots, x_{(n+1)(k-1)+1}\right)=\left(\prod_{j=1}^{k-1} x_{j}^{d^{j}}\right) x_{k}^{d^{k-1}}\left(\prod_{j=1}^{k-1} \prod_{i=1}^{n} x_{(j-1) n+i+k}^{d^{k-1-j} \cdot \alpha(i)}\right) \in C$

By substitution of monomials we obtain

$$
\begin{aligned}
& m\left(x_{1}, m_{k-1}\left(x_{2}, \ldots, x_{(n+1)(k-1)+2}\right), x_{(n+1)(k-1)+3}, \ldots, x_{(n+1) k+1}\right) \\
& \left.\left.=x_{1}^{d}\left(\left(\prod_{j=1}^{k-1} x_{j+1}^{d^{j}}\right) x_{k+1}^{d^{k-1}}\left(\prod_{j=1}^{k-1} \prod_{i=1}^{n} x_{(j-1) n+i+k+1}^{d^{k-1-j} \cdot \alpha(i)}\right)\right)\right)^{d} \prod_{i=1}^{n} x_{(n+1)(k-1)+i+2}^{\alpha(i)}\right) \\
& =x_{1}^{d}\left(\prod_{j=1}^{k-1} x_{j+1}^{d^{j+1}}\right) x_{k+1}^{d^{k}}\left(\prod_{j=1}^{k-1} \prod_{i=1}^{n} x_{(j-1) n+i+k+1}^{d^{k-j} \cdot \alpha(i)}\right)\left(\prod_{i=1}^{n} x_{(k-1) n+i+k+1}^{\alpha(i)}\right) \\
& =\left(\prod_{j=1}^{k} x_{j}^{d^{j}}\right) x_{k+1}^{d^{k}}\left(\prod_{j=1}^{k} \prod_{i=1}^{n} x_{(j-1) n+i+k+1}^{d^{k-j} \cdot \alpha(i)}\right) \in C,
\end{aligned}
$$

which concludes the induction step. Let $d^{\prime}:=\operatorname{gcd}(d, q-1)$ and let $d^{\prime \prime}:=\frac{q-1}{d^{\prime}}$. Moreover, let $k^{\prime}:=\phi\left(d^{\prime \prime}\right) \cdot(q-1)+1$, where $\phi$ denotes Euler's totient function.

Case 1 . We assume $n>0$ : In $m_{k^{\prime}}$ we set $x_{1}, \ldots, x_{k^{\prime}}$, to $x_{1}$ and $x_{k^{\prime}+1}$ to $x_{2}$. Now we set the last $n$ variables. To this end, the $x_{i}$ with $i>\left(k^{\prime}-1\right) n+k^{\prime}+1$ are set by $x_{\left(k^{\prime}-1\right) n+k^{\prime}+1+l}:=x_{2+l}$ for all $l \in\{1, \ldots, n\}$. For those $i$ with $k^{\prime}+1<i \leq\left(k^{\prime}-1\right) n+k^{\prime}+1$, we set $x_{i}:=x_{3+n}$. Hence, we get

$$
m^{\prime}=x_{1}^{\sum_{j=1}^{k^{\prime}} d^{j}} x_{2}^{d^{k^{\prime}}} x_{3+n}^{\sum_{j=1}^{k^{\prime}-1} \sum_{i=1}^{n} d^{k^{\prime}-j} \cdot \alpha(i)} \prod_{i=1}^{n} x_{2+i}^{\alpha(i)} \in C
$$

Since $q-1$ is square-free, we have $\operatorname{gcd}\left(d, \frac{q-1}{d^{\prime}}\right)=1$, and thus $d^{\phi\left(d^{\prime \prime}\right)} \equiv_{d^{\prime \prime}} 1$, since $d^{\prime \prime}=\frac{q-1}{d^{\prime}}$. Hence $d^{\phi\left(d^{\prime \prime}\right)+1} \equiv_{q-1} d$, and thus if $k, l \in \mathbb{N}$ with $k \equiv_{\phi\left(d^{\prime \prime}\right)} l$, then $d^{k} \equiv_{q-1} d^{l}$. Therefore we have $\overline{d^{k^{\prime}}}=\overline{d^{\phi\left(d^{\prime \prime}\right) \cdot(q-1)+1}}=\bar{d}, \overline{\sum_{j=1}^{k^{\prime}} d^{j}}=$ 


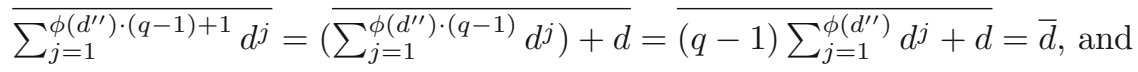

$$
\begin{aligned}
\overline{\sum_{j=1}^{k^{\prime}-1} \sum_{i=1}^{n} d^{k^{\prime}-j} \cdot \alpha(i)} & =\overline{\overline{\sum_{i=1}^{n} \alpha(i) \sum_{j=1}^{k^{\prime}-1} d^{j}}=\overline{\sum_{i=1}^{n} \alpha(i)(q-1) \sum_{j=1}^{\phi\left(d^{\prime \prime}\right)} d^{j}}} \\
& =(q-1) \underbrace{\sum_{i=1}^{n} \alpha(i) \sum_{j=1}^{\phi\left(d^{\prime \prime}\right)} d^{j}}_{\neq 0}=q-1 .
\end{aligned}
$$

Hence we have $x_{1}^{d} x_{2}^{d}\left(\prod_{i=1}^{n} x_{2+i}^{\alpha(i)}\right) x_{3+n}^{q-1} \in C$, and thus the statement is true for $n>0$.

Case 2. We assume that $n=0$ : Now in $m_{k^{\prime}}$ we set $x_{1}, \ldots, x_{k^{\prime}-1}$ to $x_{3}, x_{k^{\prime}}$ to $x_{1}$, and $x_{k^{\prime}+1}$ to $x_{2}$. Then $x_{1}^{d^{k^{\prime}}} x_{2}^{d^{k^{\prime}}} x_{3}^{a} \in C$ where $a=\sum_{i=1}^{k^{\prime}-1} d^{i}$. Similarly as in Case 1, we have $\overline{d^{k^{\prime}}}=\bar{d}$ and $\bar{a}=\overline{\sum_{i=1}^{k^{\prime}-1} d^{i}}=\overline{\sum_{i=1}^{\phi\left(d^{\prime \prime}\right) \cdot(q-1)} d^{i}}=$ $\overline{(q-1) \sum_{i=1}^{\phi\left(d^{\prime \prime}\right)} d^{i}}=q-1$.

Lemma 4.2. Let $q$ be a prime power such that $q-1$ is square-free. Let $C$ be a 0preserving semi-affine clone with respect to $\left(\mathbb{Z}_{q-1},+,-, 0\right)$. Then the function

$$
\begin{aligned}
f:\left\{G \in \mathbb{M}_{q} \mid \varphi(G)=C\right\} & \rightarrow\left\{G \cap \mathbb{F}_{q}\left[x_{1}, \ldots, x_{q}\right] \mid G \in \mathbb{M}_{q}, \varphi(G)=C\right\} \\
G & \mapsto f(G):=G \cap \mathbb{F}_{q}\left[x_{1}, \ldots, x_{q}\right]
\end{aligned}
$$

is injective.

Proof. We suppose that $G^{\prime} \nsubseteq G$ and $\varphi(G)=\varphi\left(G^{\prime}\right)$. In order to prove the statement, we show that $f\left(G^{\prime}\right) \nsubseteq f(G)$. If $q=2$, we have by Proposition 3.1 that $G=\left\langle\left\{x_{1}\right\}\right\rangle$ and $G^{\prime}=\left\langle\left\{x_{1} x_{2}\right\}\right\rangle$. Then $G^{\prime} \cap \mathbb{F}_{q}\left[x_{1}, x_{2}\right] \not \subseteq G \cap \mathbb{F}_{q}\left[x_{1}, x_{2}\right]$. Let $q>2$ and let $m^{\prime} \in G^{\prime} \backslash G$. By permuting variables and the closure under equivalent monomials we assume that $m^{\prime}$ is of the form $m^{\prime}=\left(\prod_{i=1}^{n} x_{i}^{\alpha(i)}\right)\left(\prod_{j=1}^{l_{1}} x_{n+j}^{q-1}\right)$ with $n, l_{1} \geq 0$ and for all $i \leq n, \alpha(i) \in\{1, \ldots, q-2\}$. Since $\varphi(G)=\varphi\left(G^{\prime}\right)$, there is a monomial $\left(\prod_{i=1}^{n+l_{1}} x_{i}^{\beta(i)}\right) \in G$ where $\beta(i) \equiv_{q-1} \alpha(i)$ for each $i \leq n$ and $\beta(i) \equiv_{q-1} 0$ for each $i \in\left\{n+1, \ldots, n+l_{1}\right\}$. Since $G$ is closed under equivalent monomials, we have $\left(\prod_{i=1}^{n} x_{i}^{\alpha(i)}\right)\left(\prod_{j=1}^{l_{1}} x_{n+j}^{\overline{\beta(n+j)}}\right) \in G$. Permuting variables yields $m=\left(\prod_{i=1}^{n} x_{i}^{\alpha(i)}\right)\left(\prod_{j=1}^{l_{2}} x_{n+j}^{q-1}\right) \in G$ for some $l_{2} \in \mathbb{N}_{0}, l_{2}<l_{1}$.

Case 1. We assume that $n=0$. By Lemma 2.4 a monomial clone $D$ contains $x_{1}^{q-1} x_{2}^{q-1}$ if and only if for all $l \in \mathbb{N}, \prod_{i=1}^{l} x_{i}^{q-1}$ lies in $D$. Since $l_{1}, l_{2}>$ 0 and $m^{\prime} \in G^{\prime} \backslash G$, we have $x_{1}^{q-1} x_{2}^{q-1} \in G^{\prime} \backslash G$, and thus $G^{\prime} \cap \mathbb{F}_{q}\left[x_{1}, \ldots, x_{q}\right] \nsubseteq$ $G \cap \mathbb{F}_{q}\left[x_{1}, \ldots, x_{q}\right]$. Hence $f\left(G^{\prime}\right) \not \subset f(G)$.

Case 2. We assume that $n>0$. By Lemma 2.4 we have that a monomial $\left(\prod_{i=1}^{n} x_{i}^{\alpha(i)}\right) x_{n+1}^{q-1}$ lies in a monomial clone $D$ if and only if for all $l \in \mathbb{N}_{0}$, $\left(\prod_{i=1}^{n} x_{i}^{\alpha(i)}\right)\left(\prod_{j=1}^{l} x_{n+j}^{q-1}\right)$ lies in $D$. Since $m \in G$ and $m^{\prime} \in G^{\prime} \backslash G$, we have $\left(\prod_{i=1}^{n} x_{i}^{\alpha(i)}\right) x_{n+1}^{q-1} \in G^{\prime} \backslash G$ and $m=\prod_{i=1}^{n} x_{i}^{\alpha(i)}$. If $n \geq q$, then there occurs one of the exponents of $m=\prod_{i=1}^{n} x_{i}^{\alpha(i)}$ at least twice, and by permuting variables 
and Lemma 4.1 we get that $m \cdot x_{n+1}^{q-1} \in G$, a contradiction. If $n<q$, we have $\left(\prod_{i=1}^{n} x_{i}^{\alpha(i)}\right) x_{n+1}^{q-1} \in G^{\prime} \cap \mathbb{F}_{q}\left[x_{1}, \ldots, x_{q}\right] \backslash G$. Hence $f\left(G^{\prime}\right) \nsubseteq f(G)$.

Proposition 4.3. Let $q$ be a prime power. Then $\varphi$ is a surjective map from the monomial clones on $\mathbb{F}_{q}$ to the set of 0 -preserving semi-affine clones with respect to $\left(\mathbb{Z}_{q-1},+,-, 0\right)$. Furthermore the following hold: Let $C$ be a 0 -preserving semi-affine clone with respect to $\left(\mathbb{Z}_{q-1},+,-, 0\right)$.

(1) If $C=\langle\emptyset\rangle_{\mathbb{Z}_{q-1}}$, then $\left\{G \in \mathbb{M}_{q} \mid \varphi(G)=C\right\}=\left\{\left\langle\left\{x_{1}\right\}\right\rangle,\left\langle\left\{x_{1} x_{2}^{q-1}\right\}\right\rangle\right\}$.

(2) If for all $G \in \mathbb{M}_{q}$ with $\varphi(G)=C$ we have $x_{1} x_{2}^{q-1} \in G$, then we have that $\left|\left\{G \in \mathbb{M}_{q} \mid \varphi(G)=C\right\}\right|=1$.

(3) If $q-1$ is square-free, then $\left\{G \in \mathbb{M}_{q} \mid \varphi(G)=C\right\}$ is finite.

Proof. Let $C$ be a 0 -preserving semi-affine clone w.r.t. $\left(\mathbb{Z}_{q-1},+,-, 0\right)$. We define $G^{\prime}$ as

$$
G^{\prime}:=\left\{\prod_{i=1}^{n} x_{i}^{r(i)} \mid\left(\left(y_{1}, \ldots, y_{n}\right) \mapsto \sum_{i=1}^{n}[r(i)] y_{i}\right) \in C, \exists i \leq n: r(i) \neq 0\right\} .
$$

The condition $(\exists i \leq n: r(i) \neq 0)$ is required to exclude $x_{1}^{0}=1$ as a monomial in $G^{\prime}$.

First, we show that $\varphi$ is surjective. We have that $C$ is a 0 -preserving semi-affine clone with respect to $\left(\mathbb{Z}_{q-1},+,-, 0\right)$. We have that $\left\langle\left\{x_{1}\right\}\right\rangle \subseteq G^{\prime}$, $G^{\prime}$ is closed under substitution of monomials, and $G^{\prime}$ is closed under equivalent monomials. Hence, $G^{\prime}$ is a monomial clone on $\mathbb{F}_{q}$. Furthermore, we have $\varphi\left(G^{\prime}\right)=C$ and $G^{\prime}$ is the largest $G \in \mathbb{M}_{q}$ such that $\varphi(G)=C$.

Now we show the second part of the statement and start with Item (1). If $C=\langle\emptyset\rangle_{\mathbb{Z}_{q-1}}$, then $C$ is the clone of projections. Then we see by Lemma 2.4 that $\left\{G \in \mathbb{M}_{q} \mid \varphi(G)=C\right\}=\left\{\left\langle\left\{x_{1}\right\}\right\rangle,\left\langle\left\{x_{1} x_{2}^{q-1}\right\}\right\rangle\right\}$. For Item (2) we observe the following: Let $G \in \mathbb{M}_{q}$ with $\varphi(G)=C$. Now let $m=\prod_{i=1}^{n} x_{i}^{\alpha(i)} \in G$. Since $m^{\prime}=x_{1} x_{2}^{q-1} \in G$ we get $m^{\prime}\left(m, x_{n+1}\right)=\left(\prod_{i=1}^{n} x_{i}^{\alpha(i)}\right) x_{n+1}^{q-1} \in G$. Now we see by Lemma 2.4 and by identifying variables that $G=G^{\prime}$. Now we show Item (3). There are only finitely many different non-equivalent monomials in $\mathbb{F}_{q}\left[x_{1}, \ldots, x_{q}\right]$. Therefore, we get that the co-domain of the function $f$ of Lemma 4.2 is finite. By Lemma 4.2 we know that $f$ is injective, and thus the domain $\left\{G \in \mathbb{M}_{q} \mid \varphi(G)=C\right\}$ of $f$ is finite.

Let $q$ be a prime power. Concluding this section, we note that the investigation of the lattice of monomial clones on $\mathbb{F}_{q}$ is equivalent to the problem of finding the subclones of the clone of the algebra $\left(\mathbb{F}_{q}, \cdot\right)$, which is isomorphic to $\left(\mathbb{Z}_{q-1} \cup\{-\infty\},+\right)$, where we extend the operation + on $\mathbb{Z}_{q-1}$ by $(-\infty)+b=b+(-\infty)=-\infty$ for all $b \in \mathbb{Z}_{q-1} \cup\{-\infty\}$.

\section{The top and the bottom of the lattice of monomial clones}

Let $q>2$ be a prime power. From [8] we know that the lattice of monomial clones where the clones are generated by one single binary monomial $x_{1} x_{2}^{b}$ is isomorphic to the divisor lattice of $q-1$. We already see in Figure $1 \mathrm{~b}$ for 
$q=3$ that the set $\left\{\left\langle\left\{x_{1} x_{2}^{b}\right\}\right\rangle \mid b\right.$ divides $\left.q-1\right\}$ is not equal to the interval $\left[\left\langle\left\{x_{1} x_{2}^{2}\right\}\right\rangle,\left\langle\left\{x_{1} x_{2}\right\}\right\rangle\right]$ in the lattice of monomial clones on $\mathbb{F}_{q}$. In this section we consider the top and the bottom of the lattice of monomial clones on $\mathbb{F}_{q}$ for any prime power $q>2$. We start to describe the atoms of the lattice and then we continue with the coatoms.

\subsection{Atoms}

Investigations on the atoms of the lattice of monomial clones generated by a unary function can be found in [8]. We will use one of their results in Corollary 5.2 .

Lemma 5.1. Let $q$ be a prime power and let $C \neq\left\langle\left\{x_{1}\right\}\right\rangle$ be an idempotent monomial clone on $\mathbb{F}_{q}$. Then $C$ contains $x_{1} x_{2}^{q-1}$.

Proof. Since $C \neq\left\langle\left\{x_{1}\right\}\right\rangle$, we have that there is an $n \in \mathbb{N}$ with $n \geq 2$, and there are $\alpha(1), \ldots, \alpha(n) \in \mathbb{N}$ such that $m=\prod_{i=1}^{n} x_{i}^{\alpha(i)} \in C$. Now we identify all variables $x_{i}$ where $i \geq 3$ with $x_{2}$ and get $x_{1}^{\alpha(1)} x_{2}^{\sum_{i=2}^{n} \alpha(i)} \in C$. Since $m$ is idempotent we have $\alpha(1)+\sum_{i=2}^{n} \alpha(i) \equiv_{q-1} 1$, and thus $C$ contains the monomial $x_{1}^{\overline{\alpha(1)}} x_{2}^{q-\overline{\alpha(1)}}$. By [5, Theorem 2.4] we now get $x_{1} x_{2}^{q-1} \in$ $\left\langle\left\{x_{1}^{\overline{\alpha(1)}} x_{2}^{q-\overline{\alpha(1)}}\right\}\right\rangle$.

Corollary 5.2. Let $q$ be a prime power. Then the atoms of the lattice of monomial clones on $\mathbb{F}_{q}$ are given by $\left\langle\left\{x_{1} x_{2}^{q-1}\right\}\right\rangle$ and $\left\langle\left\{x_{1}^{s}\right\}\right\rangle$ where $2 \leq s \leq q-1$ and $s^{P} \equiv_{q-1} 1$ for some prime $P$ or $s \cdot s \equiv_{q-1} s$.

Proof. Let $C$ be an atom of the lattice of monomial clones on $\mathbb{F}_{q}$. If $C$ contains a non-idempotent monomial, then, by variable identification and reduction of the exponent, $C$ contains a monomial $x_{1}^{t}$ for some $t \in \mathbb{N}$ with $2 \leq t \leq q-1$. As $C$ is an atom and $x_{1}^{t} \notin\left\langle\left\{x_{1}\right\}\right\rangle$, we have $C=\left\langle\left\{x_{1}^{t}\right\}\right\rangle$. The result now follows from [8, Lemma 3.1], where more details can be found in [7]. Now we assume that $C$ contains only idempotent monomials. Since $\left\langle\left\{x_{1}\right\}\right\rangle$ is a proper subset of $C$, we have $x_{1} x_{2}^{q-1} \in C$ by Lemma 5.1, and thus $C=\left\langle\left\{x_{1} x_{2}^{q-1}\right\}\right\rangle$.

\subsection{Coatoms}

Lemma 5.3. Let $q$ be a prime power and let $C$ be a monomial clone on $\mathbb{F}_{q}$. Let $k, l \in \mathbb{N}$. Then $x_{1} \cdots x_{1+k} \in C$ and $x_{1} \cdots x_{1+l} \in C$ if and only if $x_{1} \cdots$ $x_{1+\operatorname{gcd}(k, l)} \in C$.

Proof. By Lemma 2.7 we have $x_{1} \cdots x_{1+t \cdot \operatorname{gcd}(k, l)} \in C$ for all $t \in \mathbb{N}$, and thus the "if"-direction holds. Now we prove the "only if"-direction. Let $k^{\prime}:=\operatorname{gcd}(k, q-$ 1) and $l^{\prime}:=\operatorname{gcd}(l, q-1)$. By Lemma 2.15 we have for all $t_{1}, t_{2} \in \mathbb{N}_{0}$, that $m_{t_{1}}^{1}:=x_{1} \cdots x_{1+t_{1} \cdot k^{\prime}}$ and $m_{t_{2}}^{2}:=x_{1} \cdots x_{1+t_{2} \cdot l^{\prime}}$ lie in $C$. Let $t_{1}, t_{2} \in \mathbb{N}_{0}$ with $t_{1}>0$ or $t_{2}>0$. Then

$$
m_{t_{1}}^{1}\left(x_{1}, \ldots, x_{t_{1} \cdot k^{\prime}}, m_{t_{2}}^{2}\left(x_{1+t_{1} \cdot k^{\prime}}, \ldots, x_{1+t_{1} \cdot k^{\prime}+t_{2} \cdot l^{\prime}}\right)\right)=x_{1} \cdots x_{1+t_{1} \cdot k^{\prime}+t_{2} \cdot l^{\prime}} \in C \text {. }
$$

We proceed similar as in Lemma 2.15. By identifying variables we get that the monomial $x_{1} x_{2}^{t_{1} \cdot k^{\prime}+t_{2} \cdot l^{\prime}}$ lies in $C$. There are $u_{1}, u_{2} \in \mathbb{Z}$ such that $u_{1} \cdot k^{\prime}+u_{2} \cdot l^{\prime}=\operatorname{gcd}(k, l, q-1)$, since $k^{\prime}=\operatorname{gcd}(k, q-1)$ and $l^{\prime}=\operatorname{gcd}(l, q-$ 
1). Hence there are $t_{1}^{\prime}, t_{2}^{\prime} \in \mathbb{N}$ such that $\overline{t_{1}^{\prime} \cdot k^{\prime}+t_{2}^{\prime} \cdot l^{\prime}}=\operatorname{gcd}(k, l, q-1)$. This means that $x_{1} x_{2}^{\operatorname{gcd}(k, l, q-1)} \in C$, and by identifying variables we get $x_{1}^{1+\operatorname{gcd}(k, l, q-1)} \in C$. We have $x_{1} \cdots x_{1+k} \in C$ and thus we get by Lemma 2.11 that $x_{1} \cdots x_{q} \in C$. Since $x_{1} \cdots x_{q} \in C$ and $x_{1}^{1+\operatorname{gcd}(k, l, q-1)} \in C$ we get by Corollary 2.13 that $x_{1} \cdots x_{1+\operatorname{gcd}(k, l, q-1)} \in C$. By Lemma 2.7 we get now that $x_{1} \cdots x_{1+t \cdot \operatorname{gcd}(k, l, q-1)} \in C$ for all $t \in \mathbb{N}$, and thus we have $x_{1} \cdots x_{1+\operatorname{gcd}(k, l)}$ $\in C$.

Theorem 5.4 shows that the lattice between $\left\langle\left\{x_{1} \cdots x_{q}\right\}\right\rangle$ and $\left\langle\left\{x_{1} x_{2}\right\}\right\rangle$ is antiisomorphic to the divisor lattice of $q-1$. We denote the lattice of (positive) divisors of $q-1$ by $\mathcal{D}(q-1)$ and the lattice $\left(\left[\left\langle\left\{x_{1} \cdots x_{q}\right\}\right\rangle,\left\langle\left\{x_{1} x_{2}\right\}\right\rangle\right], \vee, \cap\right)$ by $\mathcal{X}(q)$.

Theorem 5.4. Let $q$ be a prime power, and let $\mathcal{C}: \mathcal{D}(q-1) \rightarrow \mathcal{X}(q), \mathcal{C}(a):=$ $\left\langle\left\{x_{1} \cdots x_{a+1}\right\}\right\rangle$. Let $a, b \in \mathcal{D}(q-1)$. Then $b \mid a$ if and only if $\mathcal{C}(a) \subseteq \mathcal{C}(b)$. Furthermore, $\mathcal{C}$ is surjective, and thus $\mathcal{C}$ is a lattice isomorphism from $\mathcal{D}(q-1)$ to $\mathcal{X}(q)$.

Proof. The claim is obvious for $q=2$, so we assume $q \geq 3$. If $b \mid a$, there exists $t \leq q-1$ such that $t \cdot b=a$. Then we get by Lemma 2.7, that $x_{1} \cdots x_{1+a}=x_{1} \cdots x_{1+t \cdot b} \in \mathcal{C}(b)$, and thus $\mathcal{C}(a) \subseteq \mathcal{C}(b)$. Now we assume $\mathcal{C}(a) \subseteq \mathcal{C}(b)$. By Lemma 2.15 we have that $\left\langle\left\{x_{1} \cdots x_{1+b}\right\}\right\rangle$ precisely contains all monomials where the sum of exponents is congruent to 1 modulo $b$. Therefore, we have that $b$ is the smallest natural number $k \in \mathbb{N}$ such that $x_{1} \cdots x_{1+k} \in \mathcal{C}(b)$. By Lemma 5.3 we get $x_{1} \cdots x_{1+\operatorname{gcd}(a, b)} \in \mathcal{C}(b)$. Since $b$ is the smallest element $k \in \mathbb{N}$ such that $x_{1} \cdots x_{1+k} \in \mathcal{C}(b)$, we have $\operatorname{gcd}(a, b)=b$, and thus $b \mid a$. Hence $\mathcal{C}$ is an order embedding.

To prove surjectivity, we show the following: If $C$ is a monomial clone on $\mathbb{F}_{q}$ that contains $x_{1} \cdots x_{q}$, then there exists an $a \in \mathbb{N}$ with $a \mid q-1$ such that $C=\mathcal{C}(a)$. First, we show that for all monomials $m$ there exists an $a \in \mathbb{N}$ with $a \mid q-1$ such that $\left\langle\left\{m, x_{1} \cdots x_{q}\right\}\right\rangle=\mathcal{C}(a)$. Let $m=x_{1}^{\alpha(1)} \cdots x_{n}^{\alpha(n)}$. Then $x_{1}^{\alpha} \in\left\langle\left\{m, x_{1} \cdots x_{q}\right\}\right\rangle$ where $\alpha=\overline{\sum_{i=1}^{n} \alpha(i)}$. If $\alpha=q-1$, then $x_{1}^{q-1}$ and $x_{1} \cdots x_{q}$ lie $C$, so we have $x_{1} x_{2} x_{3}^{q-1} \cdots x_{q}^{q-1} \in C$, and thus Lemma 2.2 yields $x_{1} x_{2} \in\left\langle\left\{m, x_{1} \cdots x_{q}\right\}\right\rangle$. Hence, $\left\langle\left\{m, x_{1} \cdots x_{q}\right\}\right\rangle=\left\langle\left\{x_{1} x_{2}\right\}\right\rangle$. If $\alpha=1$, then $\left\langle\left\{m, x_{1} \cdots x_{q}\right\}\right\rangle=\left\langle\left\{x_{1} \cdots x_{q}\right\}\right\rangle=\mathcal{C}(q-1)$ by Lemma 2.14. Now we assume that $1<\alpha<q-1$. By Corollary 2.13 we get $x_{1} \cdots x_{\alpha} \in\left\langle\left\{m, x_{1} \cdots x_{q}\right\}\right\rangle$ and thus $\left\langle\left\{x_{1} \cdots x_{\alpha}, x_{1} \cdots x_{q}\right\}\right\rangle \subseteq\left\langle\left\{m, x_{1} \cdots x_{q}\right\}\right\rangle$. By Lemma 5.3 we have that $x_{1} \cdots x_{1+\operatorname{gcd}(\alpha-1, q-1)} \in\left\langle\left\{x_{1} \cdots x_{\alpha}, x_{1} \cdots x_{q}\right\}\right\rangle \subseteq\left\langle\left\{m, x_{1} \cdots x_{q}\right\}\right\rangle$. On the other hand, it follows from Lemma 2.15 that $m$ and $x_{1} \cdots x_{q}$ lie in the monomial clone $\left\langle\left\{x_{1} \cdots x_{1+\operatorname{gcd}(\alpha-1, q-1)}\right\}\right\rangle$. Hence we have $\left\langle\left\{m, x_{1} \cdots x_{q}\right\}\right\rangle=\mathcal{C}(\operatorname{gcd}(\alpha-$ $1, q-1))$. We showed that for all monomials $m$ there exists an $a \in \mathbb{N}$ with $a \mid q-1$ such that $\left\langle\left\{m, x_{1} \cdots x_{q}\right\}\right\rangle=\mathcal{C}(a)$.

Let $C$ be a monomial clone on $\mathbb{F}_{q}$ that contains $x_{1} \cdots x_{q}$. We know that for every $m \in C$ there is some $a_{m} \in \mathcal{D}(q-1)$ such that $\mathcal{C}\left(a_{m}\right)=\left\langle\left\{m, x_{1} \cdots x_{q}\right\}\right\rangle \subseteq$ $C$. The set $A=\left\{a_{m} \mid m \in C\right\} \subseteq \mathcal{D}(q-1)$ is finite, so $a:=\operatorname{gcd}(A)$ can be computed. From Lemma 5.3 it follows that $\mathcal{C}(a) \subseteq C$, and conversely for every $m \in C$ we have $m \in \mathcal{C}\left(a_{m}\right) \subseteq \mathcal{C}(a)$. Thus $C=\mathcal{C}(a)$. 
Lemma 5.5. Let $q$ be a prime power and let $C$ be a monomial clone on $\mathbb{F}_{q}$. Let $n \in \mathbb{N}$ with $n \geq 2$, and let $\alpha(1), \ldots, \alpha(n) \in \mathbb{N}$ and let $\gamma \in \mathbb{N}_{0}$. We assume that $m=x_{1}^{\alpha(1)} \cdots x_{n}^{\alpha(n)} x_{n+1}^{\gamma} \in C$. Then for all $t \in \mathbb{N}_{0}$ there is a $k:\{1, \ldots, t\} \rightarrow \mathbb{N}$ and there is a $\gamma^{\prime} \in \mathbb{N}_{0}$ such that $x_{1}^{\alpha(1)} x_{2}^{\alpha(2)^{t+1}} x_{3}^{\alpha(3)} \cdots x_{n}^{\alpha(n)} x_{n+1}^{\alpha(1) \alpha(2)^{k(1)}} x_{n+2}^{\alpha(1) \alpha(2)^{k(2)}} \cdots x_{n+t}^{\alpha(1) \alpha(2)^{k(t)}} x_{n+t+1}^{\gamma^{\prime}} \in C$.

Proof. We proceed by induction on $t \in \mathbb{N}_{0}$. If $t=0$, then $m \in C$ and the statement is true. Let $t>0$. We assume that the induction hypothesis holds for $t-1$. By the induction hypothesis we have

$$
m^{\prime}\left(x_{1}, \ldots, x_{n+t}\right)=x_{1}^{\alpha(1)} x_{2}^{\alpha(2)^{t}} x_{3}^{\alpha(3)} \cdots x_{n}^{\alpha(n)} x_{n+1}^{\beta(1)} \cdots x_{n+t-1}^{\beta(t-1)} x_{n+t}^{\gamma^{\prime}} \in C,
$$

where for all $i \leq t-1$ there is a $k(i) \in \mathbb{N}$, such that $\beta(i)=\alpha(1) \alpha(2)^{k(i)}$, and $\gamma^{\prime} \in \mathbb{N}_{0}$. By substitution of monomials we have that

$$
m^{\prime \prime}=m^{\prime}(x_{n+1}, x_{2}, \underbrace{x_{n+t+1}, \ldots, x_{n+t+1}}_{n-2}, x_{n+2}, x_{n+3}, \ldots, x_{n+t+1}) \in C,
$$

and then

$$
\begin{aligned}
& m\left(x_{1}, m^{\prime \prime}, x_{3} \ldots, x_{n}, x_{n+t+1}\right) \\
& \quad=x_{1}^{\alpha(1)} x_{2}^{\alpha(2)^{t+1}} x_{3}^{\alpha(3)} \cdots x_{n}^{\alpha(n)} x_{n+1}^{\alpha(1) \alpha(2)} x_{n+2}^{\alpha(2) \beta(1)} \cdots x_{n+t}^{\alpha(2) \beta(t-1)} x_{n+t+1}^{\gamma^{\prime \prime}} \in C,
\end{aligned}
$$

for some $\gamma^{\prime \prime} \in \mathbb{N}_{0}$. This finishes the induction step.

Lemma 5.6. Let $n \in \mathbb{N}$ with $n>1$, and let $\alpha_{1}, \ldots, \alpha_{n}, \beta \in \mathbb{N}$ be such that for all $E \subseteq\{1, \ldots, n\}$ with $|E|=n-1$, we have $\operatorname{gcd}\left(\left\{\alpha_{i} \mid i \in E\right\} \cup\{\beta\}\right)=1$. Let $k, l \in \mathbb{N}^{\{1, \ldots, n\} \times\{1 \ldots, n\}}$. Then $\operatorname{gcd}\left(\left\{\alpha_{i}^{k(i, j)} \alpha_{j}^{l(i, j)} \mid 1 \leq i, j \leq n, i<j\right\} \cup\{\beta\}\right)=1$.

Proof. By assumption, no prime divisor of $\beta$ appears in $n-1$ of the $\alpha(i)$ 's. Therefore, every prime factor of $\beta$ appears in at most $n-2$ of the $\alpha(i)$ 's, and thus does not appear in the other 2. Hence $\operatorname{gcd}\left(\left\{\alpha_{i}^{k(i, j)} \alpha_{j}^{l(i, j)} \mid i, j \leq n, i<\right.\right.$ $j\} \cup\{\beta\})=1$.

Lemma 5.7. Let $q$ be a prime power and let $C$ be a monomial clone on $\mathbb{F}_{q}$. Let $n \in \mathbb{N}$ with $n>1$ and let $\alpha(1), \ldots, \alpha(n) \in \mathbb{N}$ be such that for all $E \subseteq\{1, \ldots, n\}$ with $|E|=n-1$ we have $\operatorname{gcd}(\{\alpha(i) \mid i \in E\} \cup\{q-1\})=1$. Let $\gamma \in \mathbb{N}_{0}$. If $x_{1}^{\alpha(1)} \cdots x_{n}^{\alpha(n)} x_{n+1}^{\gamma} \in C$, then $x_{1} \cdots x_{q} \in C$.

Proof. Let $m:=\prod_{i=1}^{n^{\prime}} x_{i}^{\beta(i)}$ be a monomial. We define for all $i, j \in\{1, \ldots, n\}$ with $i<j$,

$$
E(m, i, j):=\left\{l \in \mathbb{N} \mid n<l \leq n^{\prime}, \exists e_{1}, e_{2} \in \mathbb{N}: \beta(l)=\alpha(i)^{e_{1}} \alpha(j)^{e_{2}}\right\} .
$$

Let $t \in \mathbb{N}$. We proceed by induction on $k \in \mathbb{N} \backslash\{1\}$ to show that there exist $e \in \mathbb{N}^{n}, n^{\prime} \in \mathbb{N}$ and $h=\prod_{i=1}^{n^{\prime}} x_{i}^{\beta(i)} \in C$ with $\beta(1), \ldots, \beta\left(n^{\prime}\right) \in \mathbb{N}$ such that for all $i \in\{1, \ldots, n\}$ we have $\beta(i)=\alpha(i)^{e(i)}$ and for all $i, j \in\{1, \ldots, n\}$ with $i<j$ and $(i-1) \cdot n+j \leq k$ we have $|E(h, i, j)| \geq t$.

We assume that $k=2$. Let $i^{\prime}, j^{\prime} \in\{1, \ldots, n\}$ with $i^{\prime}<j^{\prime}$ be such that $\left(i^{\prime}-1\right) \cdot n+j^{\prime} \leq k$. Then $i^{\prime}=1$ and $j^{\prime}=2$, since $n>1$ and $i^{\prime}, j^{\prime}>0$. Since $C$ contains $x_{1}^{\alpha(1)} \cdots x_{n}^{\alpha(n)} x_{n+1}^{\gamma}$, we get the desired $h$ by Lemma 5.5 . 
Now let $k>2$. By the induction hypothesis there exist $e^{\prime} \in \mathbb{N}^{n}, n^{\prime} \in$ $\mathbb{N}$ and $h^{\prime}=\prod_{i=1}^{n^{\prime}} x_{i}^{\beta(i)} \in C$ with $\beta(1), \ldots, \beta\left(n^{\prime}\right) \in \mathbb{N}$ such that for all $i \in$ $\{1, \ldots, n\}$ we have $\beta(i)=\alpha(i)^{e^{\prime}(i)}$ and for all $i, j \in\{1, \ldots, n\}$ with $i<j$ and $(i-1) \cdot n+j \leq k-1$ we have $\left|E\left(h^{\prime}, i, j\right)\right| \geq t$. If there are $i^{\prime}, j^{\prime} \in\{1, \ldots, n\}$ such that $\left(i^{\prime}-1\right) \cdot n+j^{\prime}=k$, they are uniquely determined. If $i^{\prime} \geq j^{\prime}$ we set $h:=h^{\prime}$. Now we assume that $i^{\prime}<j^{\prime}$. We permute in $h^{\prime}$ the variable $x_{1}$ with $x_{i^{\prime}}$ and the variable $x_{2}$ with $x_{j^{\prime}}$ to get $h^{\prime \prime}$. Then we get the desired $h$ by applying Lemma 5.5 to $h^{\prime \prime}$ and by permuting back the variable $x_{i^{\prime}}$ with $x_{1}$ and the variable $x_{j^{\prime}}$ with $x_{2}$. This concludes the induction step.

By setting $t:=2 \cdot q^{2}$ and by choosing $k=n^{2}$ there is a monomial $h=x_{1}^{\beta(1)} \cdots x_{n^{\prime}}^{\beta\left(n^{\prime}\right)} \in C$ such that for all $i, j \in\{1, \ldots, n\}$ with $i<j$, we have $|E(h, i, j)| \geq t=2 \cdot q^{2}$. For every $i, j \leq n$ with $i<j$, we define $A(i, j):=$ $\left\{\overline{\alpha(i)^{e_{1}} \alpha(j)^{e_{2}}} \mid e_{1}, e_{2} \in \mathbb{N}\right\}$. We have $|A(i, j)| \leq q$ for each $i, j \leq n$ with $i<j$. Let $i, j \leq n$ with $i<j$. If for all $a \in A(i, j)$, we have $\mid\{l \in \mathbb{N} \mid n<l \leq$ $\left.n^{\prime}, a=\overline{\beta(l)}\right\} \mid \leq 2 \cdot q-1$, then $|E(h, i, j)| \leq \sum_{a \in A(i, j)} \mid\{l \in \mathbb{N} \mid n<l \leq$ $\left.n^{\prime}, a=\overline{\beta(l)}\right\} \mid \leq q \cdot(2 \cdot q-1)=2 \cdot q^{2}-q<2 \cdot q^{2}$ which is a contradiction, since $|E(h, i, j)| \geq 2 \cdot q^{2}$. Therefore, for all $i, j \leq n$ with $i<j$ there is an $a(i, j) \in A(i, j)$ such that $\left|\left\{l \in \mathbb{N} \mid n<l \leq n^{\prime}, a(i, j)=\overline{\beta(l)}\right\}\right| \geq 2 \cdot q$. We define $g:=\operatorname{gcd}\left(\left\{a(i, j) \mid(i, j) \in\{1, \ldots, n\}^{2}, i<j\right\} \cup\{q-1\}\right)$. Let $I \subseteq\{1, \ldots, n\}^{2}$ be such that $\left\{a(i, j) \mid(i, j) \in\{1, \ldots, n\}^{2}, i<j\right\}=\{a(i, j) \mid(i, j) \in I\}$ and for all $(i, j),\left(i^{\prime}, j^{\prime}\right) \in I$ with $(i, j) \neq\left(i^{\prime}, j^{\prime}\right)$ we have $a(i, j) \neq a\left(i^{\prime}, j^{\prime}\right)$. Since for all $E \subseteq\{1, \ldots, n\}$ with $|E|=n-1$ we have $\operatorname{gcd}(\{\alpha(i) \mid i \in E\} \cup\{q-1\})=1$, we get by Lemma 5.6 that $g=1$. Let $\tilde{t}: I \rightarrow\{1, \ldots, q-1\}$ be such that

$$
\overline{\sum_{(i, j) \in I} \tilde{t}(i, j) a(i, j)}=g=1
$$

Since for all $i, j \leq n$ with $i<j$ we have $\left|\left\{k \leq n^{\prime} \mid \overline{\beta(k)}=a(i, j)\right\}\right| \geq 2 \cdot q$, there are two functions $X$ and $X^{\prime}$ from $I$ to the finite subsets of $\left\{x_{l} \mid l \in \mathbb{N}\right\}$ such that for all $(i, j) \in I$ we have $\{a(i, j)\}=\left\{\overline{\beta(l)} \mid l \in \mathbb{N}, x_{l} \in X(i, j)\right\}=\{\overline{\beta(l)} \mid$ $\left.l \in \mathbb{N}, x_{l} \in X^{\prime}(i, j)\right\},|X(i, j)|=\left|X^{\prime}(i, j)\right|=\tilde{t}(i, j)$ and $X(i, j) \cap X^{\prime}(i, j)=\emptyset$. We mention that for $\left(i_{1}, j_{1}\right) \neq\left(i_{2}, j_{2}\right)$, the sets $X\left(i_{1}, j_{1}\right), X^{\prime}\left(i_{1}, j_{1}\right), X\left(i_{2}, j_{2}\right)$ and $X^{\prime}\left(i_{2}, j_{2}\right)$ are pairwise disjoint. For all $(i, j) \in I$ we set the variables of $X(i, j)$ to $x_{1}$, and we set the variables of $X^{\prime}(i, j)$ to $x_{2}$. All other variables of $h$ with exponent not equal to zero are set to $x_{3}$. Now we have $x_{1}^{S} x_{2}^{T} x_{3}^{\gamma} \in C$, where $\gamma \in \mathbb{N}_{0}$ and

$$
S:=\sum_{(i, j) \in I}\left(\sum_{x_{l} \in X(i, j)} \beta(l)\right), \text { and } T:=\sum_{(i, j) \in I}\left(\sum_{x_{l} \in X^{\prime}(i, j)} \beta(l)\right) .
$$

For all $(i, j) \in I$ we have

$$
\sum_{x_{l} \in X(i, j)} \overline{\beta(l)}=\sum_{x_{l} \in X(i, j)} a(i, j)=\tilde{t}(i, j) \cdot a(i, j)
$$


and

$$
\sum_{x_{l} \in X^{\prime}(i, j)} \overline{\beta(l)}=\sum_{x_{l} \in X^{\prime}(i, j)} a(i, j)=\tilde{t}(i, j) \cdot a(i, j),
$$

hence $\bar{S}=\bar{T}=\overline{\sum_{(i, j) \in I} \tilde{t}(i, j) a(i, j)}=g=1$. Since $C$ is closed under equivalent monomials, we have $x_{1}^{g} x_{2}^{g} x_{3}^{\gamma}=x_{1} x_{2} x_{3}^{\gamma} \in C$. Now Lemma 2.9 yields that $x_{1} \cdots x_{q}$ lies in $C$.

Let $q>2$ be a prime power, let $l \in \mathbb{N}$ and let $P_{1}<\ldots<P_{l}$ be the primes that divide $q-1$. Let $D \subseteq\{1, \ldots, l\}$ be nonempty. Now we define for $D$ the monomial clone $\mathcal{S}_{q}(D)$ by $\left\langle\left\{x_{1}^{t}, x_{1}^{P_{i}} \cdots x_{n}^{P_{i}}, x_{1} x_{2}^{T} \mid n, t \in \mathbb{N}, i \in D\right\}\right\rangle$, where $T$ is an abbreviation of $\prod_{t \in D} P_{t}$.

Lemma 5.8. Let $q>2$ be a prime power, let $l \in \mathbb{N}$ and let $P_{1}<\ldots<P_{l}$ be the primes that divide $q-1$. Let $D \subseteq\{1, \ldots, l\}$ be nonempty. Let $n \in \mathbb{N}$. Then for all $\alpha(1), \ldots, \alpha(n) \in \mathbb{N}$ we have that $x_{1}^{\alpha(1)} \cdots x_{n}^{\alpha(n)} \in \mathcal{S}_{q}(D)$ if and only if there exists $i \in D$ such that for all $j \leq n$ we have that $P_{i}$ divides $\alpha(j)$, or $\mid\left\{i \in \mathbb{N} \mid i \leq n, \prod_{t \in D} P_{t}\right.$ divides $\left.\alpha(i)\right\} \mid \geq n-1$.

Proof. Let $T:=\prod_{t \in D} P_{t}$. Now we define

$$
\begin{aligned}
C:= & \left\{\prod_{i=1}^{n} x_{i}^{\alpha(i)} \mid n \in \mathbb{N}, \exists j \leq n: \alpha(j) \neq 0,\left(\exists i \in D \forall j \leq n: \alpha(j) \equiv_{P_{i}} 0\right)\right. \\
& \left.\vee\left(\exists j \leq n \forall i \in\{1, \ldots, n\} \backslash\{j\}: \alpha(i) \equiv_{T} 0\right)\right\} .
\end{aligned}
$$

We show that

$$
C \text { is a monomial clone on } \mathbb{F}_{q} \text {. }
$$

(Claim 1)

We easily see that $\left\langle\left\{x_{1}\right\}\right\rangle \subseteq C$, and $C$ is closed under equivalent monomials, since for all $i \leq l, P_{i}$ divides $q-1$. Now we show that $C$ is closed under substitution of monomials. Let $m=\prod_{i=1}^{n} x_{i}^{\alpha(i)} \in C$ and let $m_{1}, \ldots, m_{n} \in C$. Now we show that $m^{\prime}:=m\left(m_{1}, \ldots, m_{n}\right)$ lies in $C$. Now let $n^{\prime} \in \mathbb{N}$ and let $\beta(1), \ldots, \beta\left(n^{\prime}\right) \in \mathbb{N}_{0}$ be such that $\prod_{i=1}^{n^{\prime}} x_{i}^{\beta(i)}=m^{\prime}$.

Case 1: There exists an $i \in D$ such that for all $j \leq n$ we have $\alpha(j) \equiv_{P_{i}} 0$ : Then we see that for all $j \leq n^{\prime}$ we have $\beta(j) \equiv_{P_{i}} 0$, and thus $m^{\prime} \in C$.

Case 2: There exists $j \leq n$ such that for all $i \in\{1, \ldots, n\} \backslash\{j\}$ we have $\alpha(i) \equiv_{T} 0$ : Let $n_{j} \in \mathbb{N}$ and let $\gamma(1), \ldots, \gamma\left(n_{j}\right) \in \mathbb{N}$ be such that $\prod_{i=1}^{n_{j}} x_{i}^{\gamma(i)}=$ $m_{j}$.

Case 2a: There exists an $i \in D$ such that for all $j \leq n_{j}$ we have $\gamma(j) \equiv_{P_{i}} 0$ : We see that for all $j \leq n^{\prime}$ we have $\beta(j) \equiv_{P_{i}} 0$, since $P_{i}$ divides $T$, and thus $m^{\prime} \in C$.

Case 2b: There exists $j^{\prime} \leq n_{j}$ such that for all $i \in\left\{1, \ldots, n_{j}\right\} \backslash\left\{j^{\prime}\right\}$ we have $\gamma(i) \equiv_{T} 0$ : We see that for all $i \in\left\{1, \ldots, n^{\prime}\right\} \backslash\left\{j^{\prime}\right\}$ we have $\beta(i) \equiv_{T} 0$, and thus $m^{\prime} \in C$.

Hence $C$ is closed under substitution of monomials, and thus Claim 1 holds. 
The next goal is to show that

$$
\mathcal{S}_{q}(D)=C \text {. }
$$

" $\subseteq$ " is clear since the generators of $\mathcal{S}_{q}(D)$ lie in $C$. For " $\supseteq$ " we observe the following. Let $m=\prod_{i=1}^{n} x_{i}^{\alpha(i)} \in C$. We show that $m \in \mathcal{S}_{q}(D)$. This is certainly true for $n=1$, so assume $n \geq 2$. Let $I:=\{i \in \mathbb{N} \mid i \leq n, \alpha(i)=0\}$. We have $J:=\{1, \ldots, n\} \backslash I \neq \emptyset$. Let $m^{\prime}:=\prod_{i \in J} x_{i}^{\alpha(i)} \prod_{i \in I} x_{i}^{q-1}$. Now let $\beta(1), \ldots, \beta(n) \in \mathbb{N}$ be such that for all $i \in J$ we have $\beta(i)=\alpha(i)$ and for all $i \in I$ we have $\beta(i)=q-1$. Then $m^{\prime}=\prod_{i=1}^{n} x_{i}^{\beta(i)}$. Now we show that $m^{\prime} \in \mathcal{S}_{q}(D)$.

Case 1. There exists an $i \in D$ such that for all $j \leq n$ we have $\alpha(j) \equiv_{P_{i}} 0$ : Since $q-1 \equiv_{P_{i}} 0$ we have for all $j \leq n$ that $\beta(j) \equiv_{P_{i}} 0$ : We easily see that $m^{\prime} \in\left\langle\left\{x_{1}^{t}, x_{1}^{P_{i}} \cdots x_{n}^{P_{i}} \mid t \in \mathbb{N}\right\}\right\rangle \subseteq \mathcal{S}_{q}(D)$.

Case 2. There exists $j \leq n$ such that for all $i \in\{1, \ldots, n\} \backslash\{j\}$ we have $\alpha(i) \equiv_{T} 0$ : Since $q-1 \equiv_{T} 0$ we have for all $i \in\{1, \ldots, n\} \backslash\{j\}$ that $\beta(i) \equiv_{T} 0$. We see by Lemma 2.7 and by permuting variables that $m^{\prime} \in\left\langle\left\{x_{1}^{t}, x_{1} x_{2}^{T} \mid t \in\right.\right.$ $\mathbb{N}\}\rangle \subseteq \mathcal{S}_{q}(D)$.

Since $m^{\prime} \in \mathcal{S}_{q}(D)$, we get by Lemma 2.2 that $m \in \mathcal{S}_{q}(D)$, and thus Claim 2 holds.

Lemma 5.9. Let $q>2$ be a prime power, let $l \in \mathbb{N}$ and let $P_{1}<\ldots<P_{l}$ be the primes that divide $q-1$. Let $C$ be a monomial clone on $\mathbb{F}_{q}$. We assume that for all nonempty $D \subseteq\{1, \ldots, l\}, C \nsubseteq \mathcal{S}_{q}(D)$. Then $C$ contains the monomial $x_{1} \cdots x_{q}$.

Proof. If for all $m \in C$ of width greater than 1 there exists an $i \leq l$ such that $P_{i}$ divides all exponents of $m$, then we have by Lemma 5.8 that $C \subseteq \mathcal{S}_{q}(\{1, \ldots, l\})$, which is a contradiction to our assumption that $C \nsubseteq \mathcal{S}_{q}(\{1, \ldots, l\})$. Hence, the set $C^{\prime}$ defined by

$$
\begin{aligned}
C^{\prime}:=\left\{x_{1}^{\alpha(1)} \cdots x_{n}^{\alpha(n)} \in C\right. & \mid n>1, \alpha(1), \ldots, \alpha(n) \in \mathbb{N}, \\
& \left.\forall i \leq l \exists j \leq n: P_{i} \text { does not divide } \alpha(j)\right\}
\end{aligned}
$$

is nonempty.

Let $m=x_{1}^{\alpha(1)} \cdots x_{n}^{\alpha(n)}$ be a monomial with $\alpha(1), \ldots, \alpha(n) \in \mathbb{N}$. Then we define for $i \in\{1, \ldots, n\}$,

$$
d^{\prime}(m, i):=\operatorname{gcd}\left(\{\alpha(j) \mid j \in\{1, \ldots, n\} \backslash\{i\}\} \cup\left\{P_{1} \cdots P_{l}\right\}\right) .
$$

Now let

$$
d(m):=\max \left(\left\{d^{\prime}(m, i) \mid i \in\{1, \ldots, n\}\right\}\right) .
$$

We have that $d(m)$ is equal to 1 or equal to a product of distinct primes that divides $q-1$. Now we assume that $m=x_{1}^{\alpha(1)} \cdots x_{n}^{\alpha(n)} \in C^{\prime}$ has the property that for all $m^{\prime} \in C^{\prime}$ we have

$$
d(m) \leq d\left(m^{\prime}\right)
$$

This means that $d(m)$ is minimal with respect to $\leq$. 
Case 1. We assume that $d(m)=1$ : Since $d(m)=\max \left(\left\{d^{\prime}(m, i) \mid i \in\right.\right.$ $\{1, \ldots, n\}\})$ and $P_{1}, \ldots, P_{l}$ are all primes that divide $q-1$, we have $\operatorname{gcd}(\{\alpha(i) \mid$ $i \in E\} \cup\{q-1\})=1$ for all $E \subseteq\{1, \ldots, n\}$ with $|E|=n-1$. Since $n>1$, Lemma 5.7 now yields $x_{1} \cdots x_{q} \in C$.

Case 2. Now we assume $1<d(m) \leq P_{1} \cdots P_{l}$ : We show that this assumption leads to a contradiction to the minimality of $d(m)$. Let $k \leq n$ be such that $d(m)=\operatorname{gcd}\left(\alpha(1), \ldots, \alpha(k-1), \alpha(k+1), \ldots, \alpha(n), P_{1} \cdots P_{l}\right)$. Let $D \subseteq\{1, \ldots, l\}$ be such that $d(m)=\prod_{i \in D} P_{i}$. Since $d(m)>1$, we have that $D \neq \emptyset$, and thus we have by Lemma 5.8 that $m \in \mathcal{S}_{q}(D)$. Since $m \in C^{\prime}$, we have for all $i \in D$ that $P_{i}$ does not divide $\alpha(k)$, which means $\operatorname{gcd}\left(\alpha(k), P_{i}\right)=1$ for all $i \in D$, and thus $\operatorname{gcd}(\alpha(k), d(m))=1$. We define $D^{\prime}:=\{1, \ldots, l\} \backslash D$. For all $i^{\prime} \in D^{\prime}$ there exists a $j \leq n$ with $j \neq k$ such that $\operatorname{gcd}\left(\alpha(j), P_{i^{\prime}}\right)=1$, since $d(m)=\operatorname{gcd}\left(\alpha(1), \ldots, \alpha(k-1), \alpha(k+1), \ldots, \alpha(n), P_{1} \cdots P_{l}\right)=\prod_{i \in D} P_{i}$.

Case 2a. We assume that $C^{\prime} \subseteq \mathcal{S}_{q}(D)$ : Since $C \nsubseteq \mathcal{S}_{q}(D)$, there is some $m_{1}=x_{1}^{\beta(1)} \cdots x_{n_{1}}^{\beta\left(n_{1}\right)} \in C$ with $\beta(1), \ldots, \beta\left(n_{1}\right) \in \mathbb{N}$ such that $m_{1} \notin \mathcal{S}_{q}(D)$. By substitution of monomials we get

$$
\begin{aligned}
m_{2} & :=m\left(x_{1}, \ldots x_{k-1}, m_{1}\left(x_{k}, \ldots, x_{k+n_{1}-1}\right), x_{k+n_{1}}, \ldots, x_{n+n_{1}-1}\right) \\
& =\prod_{i=1}^{k-1} x_{i}^{\alpha(i)} \prod_{j=1}^{n_{1}} x_{k+j-1}^{\alpha(k) \beta(j)} \prod_{i=k+1}^{n} x_{i+n_{1}-1}^{\alpha(i)} \in C .
\end{aligned}
$$

We show that

$$
m_{2} \notin \mathcal{S}_{q}(D) .
$$

(Claim 1)

Since $m_{1} \notin \mathcal{S}_{q}(D)$ we know by Lemma 5.8 that there are different $j_{1}, j_{2} \leq n_{1}$ such that $\operatorname{gcd}\left(\beta\left(j_{1}\right), d(m)\right)<d(m)$ and $\operatorname{gcd}\left(\beta\left(j_{2}\right), d(m)\right)<d(m)$ and for all $i \in D$ there exists a $J_{i} \leq n_{1}$ such that $\operatorname{gcd}\left(\beta\left(J_{i}\right), P_{i}\right)=1$. We have for all $i \in D$ that $\operatorname{gcd}\left(\alpha(k), P_{i}\right)=1$, and thus we have $\operatorname{gcd}\left(\alpha(k) \beta\left(j_{1}\right), d(m)\right)<$ $d(m)$ and $\operatorname{gcd}\left(\alpha(k) \beta\left(j_{2}\right), d(m)\right)<d(m)$. Furthermore, for all $i \in D$ we have $\operatorname{gcd}\left(\alpha(k) \beta\left(J_{i}\right), P_{i}\right)=1$. Since $\left\{\alpha(k) \beta(j) \mid j \leq n_{1}\right\}$ are exponents of $m_{2}$, we get by Lemma 5.8 that $m_{2} \notin \mathcal{S}_{q}(D)$, which proves Claim 1 .

Now we show that

$$
m_{2} \in C^{\prime} .
$$

(Claim 2)

Since $\alpha(1), \ldots, \alpha(n), \beta(1), \ldots, \beta\left(n_{1}\right) \in \mathbb{N}$ we have for each $j \in\left\{1, \ldots, n+n_{1}-\right.$ $1\}$ that the exponent of the variable $x_{j}$ is not equal to 0 . Let $i \in\{1, \ldots, l\}$. If $i \in D^{\prime}$, then there is a $J_{i} \leq n$ with $J_{i} \neq k$ such that $\operatorname{gcd}\left(\alpha\left(J_{i}\right), P_{i}\right)=1$. If $i \in D$, then there is a $J_{i} \leq n_{1}$ such that $\operatorname{gcd}\left(\beta\left(J_{i}\right), P_{i}\right)=1$, and thus $\operatorname{gcd}\left(\alpha(k) \beta\left(J_{i}\right), P_{i}\right)=1$. Since $\{\alpha(j) \mid j \leq n, j \neq k\} \cup\left\{\alpha(k) \beta(j) \mid j \leq n_{1}\right\}$ are exponents of $m_{2}$, we have that $m_{2} \in C^{\prime}$, which proves Claim 2.

By Claim 1 we have $m_{2} \notin \mathcal{S}_{q}(D)$ and by Claim 2 we have $m_{2} \in C^{\prime}$. This contradicts the assumption of Case 2a.

Case $2 \mathrm{~b}$. There exists $m_{1}=x_{1}^{\beta(1)} \cdots x_{n_{1}}^{\beta\left(n_{1}\right)} \in C^{\prime}$ with $m_{1} \notin \mathcal{S}_{q}(D)$ : Since $m_{1} \in C^{\prime}$ we have that $\beta(1), \ldots, \beta\left(n_{1}\right) \in \mathbb{N}$, and since $m_{1} \notin \mathcal{S}_{q}(D)$ we get by Lemma 5.8 that for all $j_{1} \leq n_{1}$ we have

$$
\operatorname{gcd}\left(d^{\prime}\left(m_{1}, j_{1}\right), d(m)\right)<d(m) .
$$


By substitution of monomials we get

$$
\begin{aligned}
m_{2} & \left(x_{1}, \ldots, x_{n_{1} \cdot n}\right) \\
& :=m\left(m_{1}\left(x_{1}, \ldots, x_{n_{1}}\right), m_{1}\left(x_{n_{1}+1}, \ldots, x_{2 \cdot n_{1}}\right), \ldots, m_{1}\left(x_{n_{1}(n-1)+1}, \ldots, x_{n_{1} \cdot n}\right)\right) \\
& =\prod_{i=1}^{n} \prod_{j=1}^{n_{1}} x_{(i-1) \cdot n_{1}+j}^{\alpha(i) \beta(j)} \in C .
\end{aligned}
$$

Since $m$ and $m_{1}$ lie in $C^{\prime}$, we have that $\operatorname{gcd}\left(\alpha(1), \ldots, \alpha(n), P_{1} \cdots P_{l}\right)=1$ and $\operatorname{gcd}\left(\beta(1), \ldots, \beta\left(n_{1}\right), P_{1} \cdots P_{l}\right)=1$. Let $j^{\prime} \leq n$ and $j_{1}^{\prime} \leq n_{1}$ be such that $d\left(m_{2}\right)=\operatorname{gcd}\left(\left\{\alpha(j) \beta\left(j_{1}\right) \mid j \leq n, j_{1} \leq n_{1},\left(j, j_{1}\right) \neq\left(j^{\prime}, j_{1}^{\prime}\right)\right\} \cup\left\{P_{1} \cdots P_{l}\right\}\right)$.

For all $j_{1} \leq n_{1}$ we have

$$
\operatorname{gcd}\left(\left\{\beta\left(j_{1}\right) \alpha(j) \mid j \leq n\right\} \cup\left\{P_{1} \cdots P_{l}\right\}\right)=\operatorname{gcd}\left(\beta\left(j_{1}\right), P_{1} \cdots P_{l}\right),
$$

since $\operatorname{gcd}\left(\alpha(1), \ldots, \alpha(n), P_{1} \cdots P_{l}\right)=1$. Hence we get for all $j_{1} \leq n_{1}$ with $j_{1} \neq j_{1}^{\prime}$ that $d\left(m_{2}\right)$ divides $\beta\left(j_{1}\right)$, and thus $d\left(m_{2}\right)$ divides $d^{\prime}\left(m_{1}, j_{1}^{\prime}\right)$. Similarly, we have for all $j \leq n$ that

$$
\operatorname{gcd}\left(\left\{\beta\left(j_{1}\right) \alpha(j) \mid j_{1} \leq n_{1}\right\} \cup\left\{P_{1} \cdots P_{l}\right\}\right)=\operatorname{gcd}\left(\alpha(j), P_{1} \cdots P_{l}\right),
$$

since $\operatorname{gcd}\left(\beta(1), \ldots, \beta\left(n_{1}\right), P_{1} \cdots P_{l}\right)=1$. Hence we get for all $j \leq n$ with $j \neq j^{\prime}$ that $d\left(m_{2}\right)$ divides $\alpha(j)$, and thus $d\left(m_{2}\right)$ divides $d^{\prime}\left(m, j^{\prime}\right)$.

This means $d\left(m_{2}\right)=\operatorname{gcd}\left(d^{\prime}\left(m, j^{\prime}\right), d\left(m_{2}\right)\right)=\operatorname{gcd}\left(d^{\prime}\left(m_{1}, j_{1}^{\prime}\right), d\left(m_{2}\right)\right)$. Since $d\left(m_{2}\right)=\operatorname{gcd}\left(d^{\prime}\left(m, j^{\prime}\right), d\left(m_{2}\right)\right)$ and $d^{\prime}\left(m, j^{\prime}\right) \leq d(m)$ we get that

$$
d\left(m_{2}\right)=\operatorname{gcd}\left(d^{\prime}\left(m, j^{\prime}\right), d\left(m_{2}\right)\right) \leq d(m) .
$$

By (P2) we have $\operatorname{gcd}\left(d^{\prime}\left(m_{1}, j_{1}^{\prime}\right), d(m)\right)<d(m)$, and since we have that $\operatorname{gcd}\left(d^{\prime}\left(m_{1}, j_{1}^{\prime}\right), d\left(m_{2}\right)\right)=d\left(m_{2}\right)$, we get that

$$
\operatorname{gcd}\left(d\left(m_{2}\right), d(m)\right)=\operatorname{gcd}\left(d\left(m_{2}\right), d^{\prime}\left(m_{1}, j_{1}^{\prime}\right), d(m)\right)<d(m) .
$$

By (5.1) and (5.2) we get that $d\left(m_{2}\right)<d(m)$. As $m_{2} \in C^{\prime}$, this contra$\operatorname{dicts}(\mathrm{P} 1)$.

This finishes the proof.

The next theorem gives a description of all coatoms of the lattice of monomial clones on $\mathbb{F}_{q}$.

Theorem 5.10. Let $q>2$ be a prime power, let $l \in \mathbb{N}$ and let $P_{1}<\ldots<P_{l}$ be the primes that divide $q-1$. Then the coatoms of the lattice of monomial clones are given by $\left\langle\left\{x_{1} \cdots x_{1+P_{i}}\right\}\right\rangle$ for $i \leq l$, and by $\mathcal{S}_{q}(D)$ for each nonempty $D \subseteq\{1, \ldots, l\}$.

Proof. By Theorem 5.4 we know that for $i \leq l,\left\langle\left\{x_{1} \cdots x_{1+P_{i}}\right\}\right\rangle$ is a coatom.

Now let $D \subseteq\{1, \ldots, l\}$ be nonempty. First, we see that the monomial clone $\mathcal{S}_{q}(D)$ is different from the monomial clones which we have described in Theorem 5.4, since by Lemma 5.8, $\mathcal{S}_{q}(D)$ does not contain a monomial with two exponents which are relatively prime to $q-1$. In particular, $x_{1} x_{2} \notin \mathcal{S}_{q}(D)$, so $\mathcal{S}_{q}(D) \subset\left\langle\left\{x_{1} x_{2}\right\}\right\rangle$.

Now we show that for different nonempty sets $D, D^{\prime} \subseteq\{1, \ldots, l\}, \mathcal{S}_{q}(D)$ and $\mathcal{S}_{q}\left(D^{\prime}\right)$ are incomparable. First, we assume $D \nsubseteq D^{\prime}$ and $D^{\prime} \nsubseteq D$. Then we 
have $t_{1} \in D \backslash D^{\prime}$ and $t_{2} \in D^{\prime} \backslash D$. We get by Lemma 5.8 that $x_{1}^{P_{t_{1}}} x_{2}^{P_{t_{1}}} \in \mathcal{S}_{q}(D)$, but $x_{1}^{P_{t_{1}}} x_{2}^{P_{t_{1}}} \notin \mathcal{S}_{q}\left(D^{\prime}\right)$. On the other hand, $x_{1}^{P_{t_{2}}} x_{2}^{P_{t_{2}}} \in \mathcal{S}_{q}\left(D^{\prime}\right)$, but $x_{1}^{P_{t_{2}}} x_{2}^{P_{t_{2}}} \notin$ $\mathcal{S}_{q}(D)$, and thus $\mathcal{S}_{q}(D)$ and $\mathcal{S}_{q}\left(D^{\prime}\right)$ are incomparable. Now let without loss of generality $D \subset D^{\prime}$, and let $t \in D^{\prime} \backslash D$. Then by Lemma 5.8, $x_{1}^{P_{t}} x_{2}^{P_{t}} \notin \mathcal{S}_{q}(D)$ and $x_{1}^{P_{t}} x_{2}^{P_{t}} \in \mathcal{S}_{q}\left(D^{\prime}\right)$, but $x_{1} x_{2}^{\prod_{i \in D} P_{i}} \in \mathcal{S}_{q}(D)$ and $x_{1} x_{2}^{\prod_{i \in D} P_{i}} \notin \mathcal{S}_{q}\left(D^{\prime}\right)$. Hence, $\mathcal{S}_{q}(D)$ and $\mathcal{S}_{q}\left(D^{\prime}\right)$ are incomparable.

The next goal is to show that for $D \subseteq\{1, \ldots, l\}$ nonempty, $\mathcal{S}_{q}(D)$ is a coatom. To this end, let $C$ be a monomial clone on $\mathbb{F}_{q}$ with $\mathcal{S}_{q}(D) \subset C$. Now we show that

$$
C=\left\langle\left\{x_{1} x_{2}\right\}\right\rangle
$$

Let $T:=\prod_{i \in D} P_{i}$. Let $m=x_{1}^{\alpha(1)} x_{2}^{\alpha(2)} \cdots x_{n}^{\alpha(n)} \in C \backslash \mathcal{S}_{q}(D)$ with exponents $\alpha(1), \ldots, \alpha(n) \in \mathbb{N}$. Then $n>1$, since $\mathcal{S}_{q}(D)$ contains all monomials of width 1. By Lemma 5.8, $m$ has at least two exponents which are not divisible by $T$, and for all $i \in D$ we have that there is a $j \leq n$ such that $P_{i}$ does not divide $\alpha(j)$. Therefore we have $\operatorname{gcd}(\alpha(1), \ldots, \alpha(n), T)=1$.

Case 1. We assume that there is a $j \leq n$ such that $\operatorname{gcd}(\alpha(j), T)=1$ : Then there is a $j^{\prime} \leq n$ with $j \neq j^{\prime}$ such that $\operatorname{gcd}\left(\alpha\left(j^{\prime}\right), T\right)<T$, since $m \notin \mathcal{S}_{q}(D)$. By permuting variables we assume without loss of generality that $j=1$ and $j^{\prime}=2$. We have that $x_{1} x_{2}^{T} \in \mathcal{S}_{q}(D) \subset C$, and thus $x_{1} x_{n+1}^{T} \in C$. Now we plug $m$ into $x_{1}$ of $x_{1} x_{n+1}^{T}$, and get $\left(\prod_{i=1}^{n} x_{i}^{\alpha(i)}\right) x_{n+1}^{T} \in C$. Since all monomials of width 1 are in $\mathcal{S}_{q}(D) \subset C$, we get for all $t_{1}, t_{2} \in \mathbb{N}$ that

$$
x_{1}^{t_{1} \cdot \alpha(1)}\left(\prod_{i=2}^{n} x_{i}^{\alpha(i)}\right) x_{n+1}^{t_{2} \cdot T} \in C .
$$

By identifying $x_{n+1}$ with $x_{1}$ we get for all $t_{1}, t_{2} \in \mathbb{N}$ that

$$
x_{1}^{t_{1} \cdot \alpha(1)+t_{2} \cdot T}\left(\prod_{i=2}^{n} x_{i}^{\alpha(i)}\right) \in C .
$$

Since $\operatorname{gcd}(\alpha(1), T)=1$, we have $x_{1} \prod_{i=2}^{n} x_{i}^{\alpha(i)} \in C$ by shifting coefficients in Bézout's identity by suitable multiples of $q-1$ and closure of $C$ under equivalent monomials. Then we get by variable identification that $x_{1} x_{2}^{\alpha(2)} x_{3}^{\gamma} \in$ $C$ for some $\gamma \in \mathbb{N}_{0}$, and thus we get by Lemma 2.7, variable permutation and identification that there exists a $\gamma^{\prime} \in \mathbb{N}_{0}$ such that $x_{1} x_{2}^{\alpha(2)} x_{3}^{\alpha(2)} x_{4}^{\gamma^{\prime}} \in C$. Since $\operatorname{gcd}(\alpha(2), T)<T$, there is an $i \in D$ such that $P_{i}$ does not divide $\alpha(2)$. We have $x_{1}^{P_{i}} x_{2}^{P_{i}} \in \mathcal{S}_{q}(D) \subset C$. Now we get by permuting variables and by substitution of monomials that

$$
x_{1}^{P_{i}} x_{2}^{P_{i}} x_{3}^{\alpha(2)} x_{4}^{\alpha(2)} x_{5}^{\gamma^{\prime}} \in C .
$$

Since all monomials of width 1 are in $\mathcal{S}_{q}(D) \subset C$, we get for all $t_{1}, t_{2} \in \mathbb{N}$ that

$$
x_{1}^{t_{1} \cdot P_{i}} x_{2}^{t_{1} \cdot P_{i}} x_{3}^{t_{2} \cdot \alpha(2)} x_{4}^{t_{2} \cdot \alpha(2)} x_{5}^{\gamma^{\prime}} \in C .
$$


By identifying $x_{3}$ with $x_{1}, x_{4}$ with $x_{2}$, and renaming $x_{5}$ to $x_{3}$, we get for all $t_{1}, t_{2} \in \mathbb{N}$ that

$$
x_{1}^{t_{1} \cdot P_{i}+t_{2} \cdot \alpha(2)} x_{2}^{t_{1} \cdot P_{i}+t_{2} \cdot \alpha(2)} x_{3}^{\gamma^{\prime}} \in C .
$$

Since $\operatorname{gcd}\left(\alpha(2), P_{i}\right)=1$, we have $x_{1} x_{2} x_{3}^{\gamma^{\prime}} \in C$ by shifting coefficients in Bézout's identity by suitable multiples of $q-1$ and closure of $C$ under equivalent monomials. Since $x_{1}^{q-1} \in C$, we get $x_{1} x_{2} x_{3}^{\gamma^{\prime} \cdot(q-1)} \in C$, and thus we get by Lemma 2.2 that $x_{1} x_{2} \in C$. Therefore $C=\left\langle\left\{x_{1} x_{2}\right\}\right\rangle$.

Case 2. Now we assume that for all $j \leq n$ we have $\operatorname{gcd}(\alpha(j), T) \neq 1$ : For each $j \leq n$, we define $I(j):=\min \left(\left\{i \in \mathbb{N} \mid i \in D, P_{i}\right.\right.$ divides $\left.\left.\alpha(j)\right\}\right)$ which is nonempty, since there is an $i \in D$ such that $P_{i}$ divides $\alpha(j)$. We have for all $j \leq n$, that $h_{j}:=x_{1}^{P_{I(j)}} x_{2}^{P_{I(j)}} \in \mathcal{S}_{q}(D) \subset C$. By substitution of monomials we get that

$$
\begin{aligned}
m^{\prime} & :=m\left(h_{1}\left(x_{1}, x_{n+1}\right), h_{2}\left(x_{2}, x_{n+2}\right) \cdots, h_{n}\left(x_{n}, x_{2 n}\right)\right) \\
& =\prod_{j=1}^{n} x_{j}^{P_{I(j)} \cdot \alpha(j)} \prod_{j=1}^{n} x_{n+j}^{P_{I(j)} \cdot \alpha(j)} \in C .
\end{aligned}
$$

We have by Lemma 5.8 that $x_{1} x_{2}^{T} x_{3}^{T} \in \mathcal{S}_{q}(D) \subset C$, and thus $x_{1} x_{2 n+1}^{T} x_{2 n+2}^{T} \in$ $C$. Now we plug $m^{\prime}$ into $x_{1}$ of $x_{1} x_{2 n+1}^{T} x_{2 n+2}^{T}$, and get

$$
\prod_{j=1}^{n} x_{j}^{P_{I(j)} \cdot \alpha(j)} \prod_{j=1}^{n} x_{n+j}^{P_{I(j)} \cdot \alpha(j)} x_{2 n+1}^{T} x_{2 n+2}^{T} \in C .
$$

Since all monomials of width 1 are in $C$, we get for all $t_{1}, \ldots, t_{n+1} \in \mathbb{N}$ that

$$
\prod_{j=1}^{n} x_{j}^{t_{j} \cdot P_{I(j)} \cdot \alpha(j)} \prod_{j=1}^{n} x_{n+j}^{t_{j} \cdot P_{I(j)} \cdot \alpha(j)} x_{2 n+1}^{t_{n+1} \cdot T} x_{2 n+2}^{t_{n+1} \cdot T} \in C .
$$

By setting $x_{1}, \ldots, x_{n}, x_{2 n+1}$ to $x_{1}$ and by setting $x_{n+1}, \ldots, x_{2 n}, x_{2 n+2}$ to $x_{2}$, we get for all $t_{1}, \ldots, t_{n+1} \in \mathbb{N}$ that

$$
x_{1}^{\sum_{j=1}^{n} t_{j} \cdot P_{I(j)} \cdot \alpha(j)+t_{n+1} \cdot T} x_{2}^{\sum_{j=1}^{n} t_{j} \cdot P_{I(j)} \cdot \alpha(j)+t_{n+1} \cdot T} \in C .
$$

We have $\operatorname{gcd}(\alpha(1), \ldots, \alpha(n), T)=1$, and for all $j \leq n, \operatorname{gcd}\left(\alpha(j), P_{I(j)}\right)=$ $P_{I(j)}$, and thus $\operatorname{gcd}\left(P_{I(1)} \cdot \alpha(1), \ldots, P_{I(n)} \cdot \alpha(n), T\right)=1$, since we have that $\operatorname{gcd}(\alpha(1), \ldots, \alpha(n), T)=1$ and $P_{I(j)} \cdot \alpha(j)$ contains the same prime factors as $\alpha(j)$, only with different multiplicities. Hence, by shifting coefficients in Bézout's lemma and closure of $C$ under equivalent monomials, $x_{1} x_{2} \in C$, and thus $C=\left\langle\left\{x_{1} x_{2}\right\}\right\rangle$.

We proved (5.3), and thus $\mathcal{S}_{q}(D)$ is a coatom.

Now we show that there are no other coatoms. Let $C$ be a coatom of the lattice of monomial clones on $\mathbb{F}_{q}$ which is different from the coatoms given in the statement. This means that for all $\emptyset \neq D \subseteq\{1, \ldots, l\}$ we have $C \nsubseteq$ $\mathcal{S}_{q}(D)$. Now Lemma 5.9 yields $x_{1} \cdots x_{q} \in C$. Since $C$ is a coatom, we get by Theorem 5.4 that $C=\left\langle\left\{x_{1} \cdots x_{1+P_{i}}\right\}\right\rangle$ for some $i \leq l$. This contradicts that $C$ is a coatom which is different from the statement. 

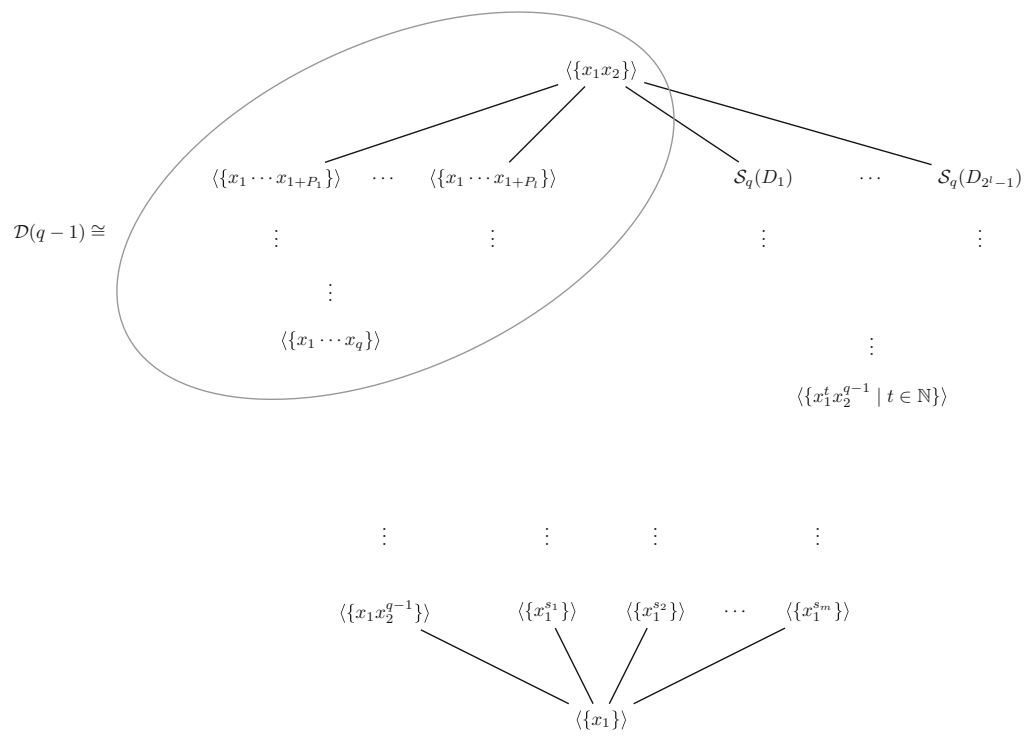

Figure 5. The frame of the lattice of monomial clones on $\mathbb{F}_{q}$

All coatoms we have found in Theorem 5.10 are different and thus we obtain the number of coatoms on $\mathbb{F}_{q}$.

Corollary 5.11. Let $q>2$ be a prime power, let $l \in \mathbb{N}$ be the number of different primes that divide $q-1$. Then there are $2^{l}-1+l$ coatoms of the lattice of monomial clones on $\mathbb{F}_{q}$.

\subsection{Summary}

We summarize this section in Figure 5. Let $q>2$ be a prime power, let $l, m, n \in \mathbb{N}$ and let $P_{1}<\ldots<P_{l}$ be all primes that divide $q-1$ and let $2 \leq s_{1}<\ldots<s_{m} \leq q-1$ be all numbers such that for all $i \leq m, s_{i}^{p} \equiv_{q-1} 1$ for some prime $p$ or $s_{i} s_{i} \equiv_{q-1} s_{i}$. Let $D_{1}, \ldots, D_{2^{l}-1}$ be all nonempty subsets of $\{1, \ldots, l\}$.

\section{Infinite chains of monomial clones}

\subsection{Monomial clones are well-partially ordered}

Consider $q \in \mathbb{N} \backslash\{1\}$. Let $S \subseteq \mathbb{N}_{0}^{q-1}$ with $(0, \ldots, 0) \in S$. We call $S$ a $q$ minor set if for all $\left(s_{1}, \ldots, s_{q-1}\right) \in S$ we have for all $t_{1}, \ldots, t_{q-1} \in \mathbb{N}_{0}$ with $s_{i}-t_{i}(q-1) \geq 0$ for all $i \leq q-1$ that $\left(s_{1}-t_{1}(q-1), \ldots, s_{q-1}-t_{q-1}(q-1)\right) \in S$. By $\mathcal{T}_{q}$ we denote the set of all $q$-minor sets.

Let $\mathbf{b}=\left(b_{1}, \ldots, b_{q-1}\right) \in\{0, \ldots, q-2\}^{q-1}$ and let $S \subseteq \mathbb{N}_{0}^{q-1}$ be a $q$-minor set. We define $M(\mathbf{b}, S)$ by $M(\mathbf{b}, S):=\left\{\left(t_{1}, \ldots, t_{q-1}\right) \in \mathbb{N}_{0}^{q-1} \mid\left(b_{1}, \ldots, b_{q-1}\right)+(q-1) \cdot\left(t_{1}, \ldots, t_{q-1}\right) \in S\right\}$. 
Let $\leq$ be the product order on $\mathbb{N}_{0}^{q-1}$. We call a subset $D \subseteq \mathbb{N}_{0}^{q-1}$ downward closed if $d \in D, d^{\prime} \in \mathbb{N}_{0}^{q-1}, d^{\prime} \leq d$ implies $d^{\prime} \in D$. By the definition of a $q$-minor set, we have that $M(\mathbf{b}, S)$ is a downward closed set for all $\mathbf{b} \in\{0, \ldots, q-2\}^{q-1}$.

Lemma 6.1. Let $S, S^{\prime} \subseteq \mathbb{N}_{0}^{q-1}$ be q-minor sets. Then $S \subseteq S^{\prime}$ if and only if $M(\mathbf{b}, S) \subseteq M\left(\mathbf{b}, S^{\prime}\right)$ for all $\mathbf{b} \in\{0, \ldots, q-2\}^{q-1}$.

Proof. The "only if"-direction obviously holds by the definition of $M$ as a preimage. For the "if"-direction we assume that $S \nsubseteq S^{\prime}$. Then there is an $s=\left(s_{1}, \ldots, s_{q-1}\right) \in S$ such that $s \notin S^{\prime}$. Now let $t_{1}, \ldots, t_{q-1} \in \mathbb{N}_{0}$ such that $0 \leq s_{i}-t_{i}(q-1) \leq q-2$. We define $\mathbf{b}:=\left(s_{1}-t_{1}(q-1), \ldots, s_{q-1}-t_{q-1}(q-1)\right)$. Then we have $\left(t_{1}, \ldots, t_{q-1}\right) \in M(\mathbf{b}, S)$, but $\left(t_{1}, \ldots, t_{q-1}\right) \notin M\left(\mathbf{b}, S^{\prime}\right)$, because $s \notin S^{\prime}$. This finishes the proof.

Let $\mathbf{b}_{1}, \ldots, \mathbf{b}_{(q-1)^{(q-1)}}$ be all elements of $\{0, \ldots, q-2\}^{q-1}$ and $\mathcal{D}\left(\mathbb{N}_{0}^{q-1}\right)$ be the set of all downward closed sets. For any $q$-minor set $S \subseteq \mathbb{N}_{0}^{q-1}$, we now define $T(S) \in\left(\mathcal{D}\left(\mathbb{N}_{0}^{q-1}\right)\right)^{(q-1)^{(q-1)}}$ by

$$
T(S):=\left(M\left(\mathbf{b}_{1}, S\right), \ldots, M\left(\mathbf{b}_{(q-1)^{(q-1)}}, S\right)\right) .
$$

Proposition 6.2. Let $q$ be a prime power. $\left(\mathcal{T}_{q}, \subseteq\right)$ is well-partially ordered.

Proof. Since the downward closed sets are the complements of the upward closed sets, we get from $\left[1\right.$, Theorem 1.1] that $\left(\mathcal{D}\left(\mathbb{N}_{0}^{q-1}\right), \subseteq\right)$ does not contain infinite antichains or infinite descending chains. Now [3, Example (4) p.195] yields that $\left(\left(\mathcal{D}\left(\mathbb{N}_{0}^{q-1}\right)\right)^{(q-1)^{(q-1)}}, \subseteq^{\prime}\right)$, where $\subseteq^{\prime}$ is the product order of $\subseteq$, does not contain infinite antichains or infinite descending chains. By Lemma 6.1 we have that $T$ is an order embedding into $\left(\left(\mathcal{D}\left(\mathbb{N}_{0}^{q-1}\right)\right)^{(q-1)^{(q-1)}}, \subseteq^{\prime}\right)$, and thus the result holds.

Let now $q$ be a prime power. Remember that $\mathbb{M}_{q}$ is the set of all monomial clones on $\mathbb{F}_{q}$, and if $C \in \mathbb{M}_{q}$, then $\bar{C}$ is the set $\left\{\prod_{i=1}^{n} x_{i}^{\alpha(i)} \in C \mid \forall i \leq n: \alpha(i) \in\right.$ $\{1, \ldots, q-1\}\}$. Let $m \in \bar{C}$. Now we define for $i \in\{1, \ldots, q-1\}, \mathcal{N}(m, i)$ as the number of exponents of $m$ equal to $i$. Let $C$ be a monomial clone. We define

$$
\phi(C):=\left\{(\mathcal{N}(m, 1), \ldots, \mathcal{N}(m, q-1)) \in \mathbb{N}_{0}^{q-1} \mid m \in \bar{C}\right\} \cup\{(0, \ldots, 0)\} .
$$

Since $C$ is closed under identifying variables, $\overline{a+(q-1) \cdot a}=\bar{a}$ for all $a \in \mathbb{N}$ and $(0, \ldots, 0) \in \phi(C)$, we have $\phi(C) \in \mathcal{T}_{q}$.

Theorem 6.3. Let $q$ be a prime power. Then $\phi$ is an order embedding from $\left(\mathbb{M}_{q}, \subseteq\right)$ to $\left(\mathcal{T}_{q}, \subseteq\right)$, and thus the lattice of monomial clones on $\mathbb{F}_{q}$ is wellpartially ordered, i.e., there is no infinite descending chain of monomial clones on $\mathbb{F}_{q}$ and there is no infinite antichain of monomial clones on $\mathbb{F}_{q}$.

Proof. We show that $C \subseteq C^{\prime}$ if and only if $\phi(C) \subseteq \phi\left(C^{\prime}\right)$. The "only if"direction holds by the definition of $\phi$. For the "if"-direction let $\phi(C) \subseteq \phi\left(C^{\prime}\right)$. Assume, without loss of generality, that $m=\prod_{i=1}^{n} x_{i}^{\alpha(i)} \in C$, where $1 \leq$ $\alpha(i) \leq q-1$ for each $i \leq n$. Then $e=\left(s_{1}, \ldots, s_{q-1}\right) \in \phi(C)$, where $s_{i}=\mathcal{N}(m, i)$ for each $i \leq q-1$. Since $\phi(C) \subseteq \phi\left(C^{\prime}\right)$, we have $e \in \phi\left(C^{\prime}\right)$. Therefore there is 
an $m^{\prime} \in \overline{C^{\prime}} \subseteq C^{\prime}$ such that $m^{\prime}=\prod_{i=1}^{n} x_{\pi(i)}^{\alpha(i)}$ for some bijective $\pi:\{1, \ldots, n\} \rightarrow$ $\{1, \ldots, n\}$. Since $C^{\prime}$ is closed under permuting variables, we have $m \in C^{\prime}$. This means $\phi$ is an order embedding from $\mathbb{M}_{q}$ to $\mathcal{T}_{q}$. By Proposition 6.2 we have that $\left(\mathcal{T}_{q}, \subseteq\right)$ is well-partially ordered, and thus the result holds.

Remark 6.4. For the embedding from monomial clones to $q$-minor sets, we have just used that a monomial clone is closed under composition with projections and closed under taking equivalent monomials. Proposition 2.13 of [15] states that for every finite set $A$, up to term equivalence, there are only countably many semi-affine algebras on $A$. Theorem 6.3 does not follow directly from this result of Á. Szendrei or its proof. On the other hand we can easily transfer the result of Theorem 6.3 to the lattice of 0 -preserving semi-affine clones with respect to a (finite) abelian group $\mathbf{A}:=(A,+,-, 0)$, by

- replacing in the definition of a $q$-minor set, $(q-1)$ by the exponent of $\mathbf{A}$,

- and counting the number of occurrences of a number $r$ below the exponent among the coefficients $r_{i}$ of $f: A^{n} \rightarrow A,\left(a_{1}, \ldots, a_{n}\right) \mapsto \sum_{i=1}^{n} r_{i} \cdot a_{i}$ (cf. the proof of [15, Proposition 2.13]).

\subsection{Infinite ascending chains}

Proposition 6.5. Let $q$ be a prime power such that there is a $k \in \mathbb{N} \backslash\{1\}$ with $k^{2} \mid q-1$. Now let $d:=\frac{q-1}{k}$. Let for each $i \in \mathbb{N}_{0}, f_{i}:=\prod_{j=1}^{k \cdot i+1} x_{j}^{d}$. Then $q \geq 5$ and $\left(\left\langle\left\{f_{i}\right\}\right\rangle\right)_{i \in \mathbb{N}_{0}}$ is an infinite ascending chain of monomial clones on $\mathbb{F}_{q}$.

Proof. Let $i \in \mathbb{N}_{0}$. We have $k \cdot d=q-1$, and thus by Lemma 2.2 we have $\left\langle\left\{f_{i}\right\}\right\rangle \subseteq\left\langle\left\{f_{i+1}\right\}\right\rangle$. Now we show that this inclusion is proper. We have $d \neq q-1$, since $k \neq 1$, and $\overline{d^{2}}=\overline{(q-1) \cdot\left(\frac{q-1}{k^{2}}\right)}=q-1$, since $k^{2} \mid q-1$. We interpret now $f_{i}$ as the $(k \cdot i+1)$-ary function which is induced by $f_{i}$. The clone generated by $f_{i}$ can be described as the set of all term functions $T^{\mathbb{F}_{q}}$ induced by some term $T$ in the language $\left\{f_{i}\right\}$ over $n^{\prime}$ variables where $n^{\prime} \in \mathbb{N}$. We now show by induction on the length of the term $T$ that if $T^{\mathbb{F}_{q}}$ is not a projection, then $T^{\mathbb{F}_{q}}$ is an $n$-ary operation on $\mathbb{F}_{q}$ for some $n \in \mathbb{N}$ which is induced by a monomial $x_{1}^{\alpha(1)} \ldots x_{n}^{\alpha(n)}$ with $\alpha(1), \ldots, \alpha(n) \in\{0,1, \ldots, q-1\}$ such that $|\{j \leq n \mid \alpha(j)=d\}| \leq k \cdot i+1$ and for each $j \leq n$ we have that $d$ divides $\alpha(j)$. If the length of the term $T$ is equal to 1 , then $T$ is a variable and thus induces a projection, whence the statement holds. Now let $T$ be of length greater than 1. Then $T=f_{i}\left(T_{1}, \ldots, T_{k \cdot i+1}\right)$ where for $j \leq k \cdot i+1, T_{j}$ is a shorter term than $T$. Let $n \in \mathbb{N}$ be the arity of $T^{\mathbb{F}_{q}}$. Now let $m=x_{1}^{\alpha(1)} \cdots x_{n}^{\alpha(n)}$ be the monomial with $\alpha(1), \ldots, \alpha(n) \in\{0,1, \ldots, q-1\}$ that induces $T^{\mathbb{F}_{q}}$. We see the following. Let $j \leq k \cdot i+1$. If $\left(T_{j}\right)^{\mathbb{F}_{q}}$ is a projection, then $\left(T_{j}^{d}\right)^{\mathbb{F}_{q}}\left(a_{1}, \ldots, a_{n}\right)=a_{i^{\prime}}^{d}$ for some $i^{\prime} \leq n$. If $\left(T_{j}\right)^{\mathbb{F}_{q}}$ is not a projection, then $\left(T_{j}^{d}\right)^{\mathbb{F}_{q}}\left(a_{1}, \ldots, a_{n}\right)=\prod_{i^{\prime} \in I} a_{i^{\prime}}^{q-1}$ for some $I \subseteq\{1, \ldots, n\}$, since by the induction hypothesis all exponents of the monomial which induces $T_{j}^{\mathbb{F} q}$ are of the form $t \cdot d$, and thus we have $t \cdot d \cdot d=t \cdot d^{2}$ where $\overline{t \cdot d^{2}}=q-1$ if $t \neq 0$. Since $d \mid q-1$, we now get that the exponents $\alpha(1), \ldots, \alpha(n)$ of $m$ are divisible by $d$. Since $d \neq q-1$, we get that the number of $d$ 's as exponent is at most the number of $T_{j}$ 's which are projections. This means that at most $k \cdot i+1$ many exponents of $m$ are equal to $d$. 
Now we have $\left\langle\left\{f_{i}\right\}\right\rangle \subset\left\langle\left\{f_{i+1}\right\}\right\rangle$, since $f_{i+1}$ contains $k \cdot(i+1)+1$ many variables with $d$ as their exponent. Hence, $\left\langle\left\{f_{0}\right\}\right\rangle \subset\left\langle\left\{f_{1}\right\}\right\rangle \subset\left\langle\left\{f_{2}\right\}\right\rangle \subset\left\langle\left\{f_{3}\right\}\right\rangle \subset \cdots$ which finishes the proof.

Example 6.6. In $\mathbb{F}_{5},\left\langle\left\{x_{1}^{2}\right\}\right\rangle \subset\left\langle\left\{x_{1}^{2} x_{2}^{2} x_{3}^{2}\right\}\right\rangle \subset\left\langle\left\{x_{1}^{2} x_{2}^{2} x_{3}^{2} x_{4}^{2} x_{5}^{2}\right\}\right\rangle \subset \cdots$ or in $\mathbb{F}_{9}$, $\left\langle\left\{x_{1}^{4}\right\}\right\rangle \subset\left\langle\left\{x_{1}^{4} x_{2}^{4} x_{3}^{4}\right\}\right\rangle \subset\left\langle\left\{x_{1}^{4} x_{2}^{4} x_{3}^{4} x_{4}^{4} x_{5}^{4}\right\}\right\rangle \subset \cdots$ are infinite ascending chains of monomial clones.

Remark 6.7. Let $q \geq 5$ be a prime power such that there is a $k \in \mathbb{N} \backslash\{1\}$ with $k^{2} \mid q-1$. We find infinite ascending chains also by the connection to semiaffine algebras: By $[15$, p. 60$]$ the clones on $\mathbb{Z}_{q-1}$, generated by $\left\{\left(x_{1}, \ldots, x_{i}\right) \mapsto\right.$ $\left.\sum_{j=1}^{i} k \cdot x_{j}\right\}$ for $i \in \mathbb{N}$, form an infinite ascending chain.

Theorem 6.8. Let $q$ be a prime power. Infinite ascending chains of monomial clones on $\mathbb{F}_{q}$ exist if and only if $q-1$ is not square-free.

Proof. If $q-1$ is not square-free, then we get infinite ascending chains by Proposition 6.5. Now we assume that $q-1$ is square-free. By Á. Szendrei (see [14] or [15, p. 61]) we know that the number of semi-affine algebras on a finite set $A$ up to term equivalence is finite if and only if $|A|$ is square-free. Since $q-1$ is square-free we have that the number of semi-affine clones with respect to $\left(\mathbb{Z}_{q-1},+,-, 0\right)$ is finite. Item $(3)$ of Proposition 4.3 now yields that the number of monomial clones on $\mathbb{F}_{q}$ is finite, and thus there do not exist infinite ascending chains.

Corollary 6.9. For a prime power $q$, the lattice of monomial clones on $\mathbb{F}_{q}$ is finite if and only if $q-1$ is square-free.

Proof. If the lattice of monomial clones on $\mathbb{F}_{q}$ is finite, then there are no infinite ascending chains. From Theorem 6.8 we then get that $q-1$ is squarefree. If the lattice of monomial clones on $\mathbb{F}_{q}$ is infinite, we get by the infinite version of Ramsey's theorem that there are infinite ascending chains, or infinite descending chains or infinite antichains. It follows from Theorem 6.3 that the lattice of monomial clones on $\mathbb{F}_{q}$ does not contain infinite descending chains and does not contain infinite antichains. Therefore the lattice of monomial clones on $\mathbb{F}_{q}$ contains an infinite ascending chain. Now Theorem 6.8 yields that $q-1$ is not square-free.

\section{Idempotent monomial clones}

Let $q$ be a prime power. In this section we investigate idempotent monomial clones on $\mathbb{F}_{q}$, this means the interval $\left[\left\langle\left\{x_{1}\right\}\right\rangle,\left\langle\left\{x_{1} \cdots x_{q}\right\}\right\rangle\right]$ (cf. Lemma 2.14).

Lemma 7.1. Let $q$ be a prime power. Let $k, l \in \mathbb{N}$ and let $m_{1}$ be a $k$-ary idempotent monomial and let $m_{2}$ be an l-ary idempotent monomial. Now let

$$
m\left(x_{1}, \ldots, x_{k \cdot l}\right):=m_{1}\left(m_{2}\left(x_{1}, \ldots, x_{l}\right), \ldots, m_{2}\left(x_{(k-1) \cdot l+1}, \ldots, x_{l \cdot k}\right)\right) .
$$

Then $\left\langle\left\{m_{1}, m_{2}\right\}\right\rangle=\langle\{m\}\rangle$. 
Proof. Let $m_{1}=\prod_{j=1}^{k} x_{j}^{\alpha(j)}$ where $\overline{\sum_{j=1}^{k} \alpha(j)}=1$, and let $m_{2}=\prod_{j=1}^{l} x_{j}^{\beta(j)}$ where $\overline{\sum_{j=1}^{l} \beta(j)}=1$. Obviously, " $\supseteq$ " holds, since $m$ is a composition of $m_{1}$ and $m_{2}$. For " $\subseteq$ " we observe the following. Using idempotency, we have

$$
m\left(x_{1}, \ldots, x_{1}, x_{2}, \ldots, x_{2}, \ldots, x_{k}, \ldots, x_{k}\right)=\prod_{j=1}^{k} x_{j}^{\alpha(j) \cdot\left(\sum_{i=1}^{l} \beta(i)\right)} \in\langle\{m\}\rangle,
$$

and thus $m_{1}=\prod_{j=1}^{k} x_{j}^{\alpha(j)} \in\langle\{m\}\rangle$, since $\overline{\sum_{i=1}^{l} \beta(i)}=1$. On the other hand we also get

$$
\begin{aligned}
m\left(x_{1}, \ldots, x_{l}, x_{1}, \ldots, x_{l}, \ldots, x_{1}, \ldots, x_{l}\right) & =\prod_{i=1}^{k}\left(\prod_{j=1}^{l} x_{j}^{\beta(j)}\right)^{\alpha(i)} \\
& =\left(\prod_{j=1}^{l} x_{j}^{\beta(j)}\right)^{\sum_{i=1}^{k} \alpha(i)} \\
& =\prod_{j=1}^{l} x_{j}^{\beta(j) \cdot\left(\sum_{i=1}^{k} \alpha(i)\right)} \in\langle\{m\}\rangle,
\end{aligned}
$$

and thus $m_{2}=\prod_{j=1}^{l} x_{j}^{\beta(j)} \in\langle\{m\}\rangle$, since $\overline{\sum_{i=1}^{k} \alpha(i)}=1$. This finishes the proof.

As a consequence of Lemma 7.1 we get the following corollary:

Corollary 7.2. Let $q$ be a prime power. A finitely generated idempotent monomial clone on $\mathbb{F}_{q}$ is singly generated.

Remark 7.3. Corollary 7.2 does not hold in general for an arbitrary monomial clone, as we can see from the case distinction for the field $\mathbb{F}_{3}$ in the proof of Proposition 3.1 where the monomial clone $\left\langle\left\{x_{1}^{2}, x_{1} x_{2}^{2}\right\}\right\rangle$ cannot be generated by one element.

Now we use again the connection of monomial clones to semi-affine algebras to show that there are only finitely many idempotent monomial clones on $\mathbb{F}_{q}$.

Proposition 7.4. Let $q$ be a prime power. The lattice of idempotent monomial clones on $\mathbb{F}_{q}$ is finite.

Proof. Let $\varphi$ be defined as in Section 4. Then $C$ is an idempotent monomial clone on $\mathbb{F}_{q}$ if and only if $\varphi(C)$ is a finite idempotent 0-preserving semi-affine clone with respect to $\left(\mathbb{Z}_{q-1},+,-, 0\right)$, since $\left(x_{1}, \ldots, x_{n}\right) \mapsto \sum_{i=1}^{n} r(i) x_{i}$ is idempotent if and only if $\sum_{i=1}^{n} r(i)=1$. By Lemma 5.1 we know that every idempotent monomial clone strictly above $\left\langle\left\{x_{1}\right\}\right\rangle$ contains $x_{1} x_{2}^{q-1}$. By Item (1) and Item (2) of Proposition 4.3 we get that $\left|\left[\left\langle\left\{x_{1}\right\}\right\rangle,\left\langle\left\{x_{1} \cdots x_{q}\right\}\right\rangle\right]\right|=\mid\left[\varphi\left(\left\langle\left\{x_{1}\right\}\right\rangle\right)\right.$, $\left.\varphi\left(\left\langle\left\{x_{1} \cdots x_{q}\right\}\right\rangle\right)\right] \mid+1$. The result follows now from [15, Corollary 2.20], which states that modulo term equivalence there are only finitely many idempotent semi-affine algebras on a fixed finite universe. 
Corollary 7.5. Let $q$ be a prime power. Every idempotent monomial clone on $\mathbb{F}_{q}$ is singly generated.

Proof. By Proposition 7.4 we get that there are no infinite ascending chains of monomial clones in $\left[\left\langle\left\{x_{1}\right\}\right\rangle,\left\langle\left\{x_{1} \cdots x_{q}\right\}\right\rangle\right]$, and thus the result follows from Corollary 7.2.

\section{Acknowledgements}

The author gratefully thanks Erhard Aichinger and Stefano Fioravanti for many hours of fruitful discussions. The author also thanks the anonymous referee for a very careful report which led to corrections of some proofs and even of some statements and thus significantly improved the quality of the paper.

Funding Open access funding provided by Austrian Science Fund (FWF).

Open Access. This article is licensed under a Creative Commons Attribution 4.0 International License, which permits use, sharing, adaptation, distribution and reproduction in any medium or format, as long as you give appropriate credit to the original author(s) and the source, provide a link to the Creative Commons licence, and indicate if changes were made. The images or other third party material in this article are included in the article's Creative Commons licence, unless indicated otherwise in a credit line to the material. If material is not included in the article's Creative Commons licence and your intended use is not permitted by statutory regulation or exceeds the permitted use, you will need to obtain permission directly from the copyright holder. To view a copy of this licence, visit http://creativecommons. org/licenses/by/4.0/.

Publisher's Note Springer Nature remains neutral with regard to jurisdictional claims in published maps and institutional affiliations.

\section{References}

[1] Aichinger, E., Aichinger, F.: Dickson's lemma, Higman's theorem and beyond: a survey of some basic results in order theory. Expo. Math. 38(4), 537-547 (2020)

[2] Aichinger, E., Mayr, P.: Polynomial clones on groups of order pq. Acta Math. Hungar. 114(3), 267-285 (2007)

[3] Aschenbrenner, M., Hemmecke, R.: Finiteness theorems in stochastic integer programming. Found. Comput. Math. 7(2), 183-227 (2007)

[4] Bulatov, A.A.: Polynomial clones containing the Mal'tsev operation of the groups $\mathbb{Z}_{p^{2}}$ and $\mathbb{Z}_{p} \times \mathbb{Z}_{p}$. Mult. Valued Log. 8(2), 193-221 (2002)

[5] Horváth., G., Kátai-Urbán, K., Szabó, C.: Monomial clones over $\mathbb{F}_{q}$. Algebra Univers. 80(2), 1-9 (2019) 
[6] Janov, J.I., Mučnik, A.A.: Existence of $k$-valued closed classes without a finite basis. Dokl. Akad. Nauk SSSR 127, 44-46 (1959)

[7] Machida, H., Pantović, J.: Monomial clones: local results and global properties. In: 2016 IEEE 46th International Symposium on Multiple-Valued Logic, IEEE Computer Soc., Los Alamitos, pp. 78-83 (2016)

[8] Machida, H., Pantović, J.: Three classes of closed sets of monomials. In: 2017 IEEE 47th International Symposium on Multiple-Valued Logic-ISMVL, IEEE, New York, pp. 100-105 (2017)

[9] Machida, H., Pantović, J.: Monomial clones over small finite fields. J. Mult. Valued Logic Soft Comput. 31(3), 271-286 (2018)

[10] Machida, H., Pinsker, M.: Some polynomials generating minimal clones. J. Mult. Valued Logic Soft Comput. 13(4-6), 353-365 (2007)

[11] Mayr, P.: Polynomial clones on squarefree groups. Int. J. Algebra Comput. 18(4), 759-777 (2008)

[12] Pöschel, R., Kalužnin, L.A.: Funktionen- und Relationenalgebren, Mathematische Monographien, vol. 15. VEB Deutscher Verlag der Wissenschaften, Berlin (1979).. (German)

[13] Post, E.L.: The Two-Valued Iterative Systems of Mathematical Logic, Annals of Mathematics Studies, no. 5. Princeton University Press, Princeton (1941)

[14] Szendrei, Á.: Clones of linear operations on finite sets, Finite algebra and multiple-valued logic (Szeged, 1979), Colloq. Math. Soc. János Bolyai, vol. 28, pp. 693-738. North-Holland, Amsterdam (1981)

[15] Szendrei, Á.: Clones in universal algebra, Séminaire de Mathématiques Supérieures [Seminar on Higher Mathematics], vol. 99. Presses de l'Université de Montréal, Montreal (1986)

Sebastian Kreinecker

Institute for Algebra

Johannes Kepler University Linz

Altenbergerstraße 69

4040 Linz

Austria

e-mail: kreinecker@algebra.uni-linz.ac.at

URL: http://www.jku.at/en/institute-for-algebra/

Received: 24 April 2020.

Accepted: 19 April 2021. 